WHOI-89-13

\title{
Satellite Data Processing System (SDPS) Users Manual V1.0
}

\author{
by \\ Michael Caruso \\ Chris Dunn \\ Woods Hole Oceanographic Institution \\ Woods Hole, Massachusetts 02543
}

May 1989

\section{Technical Report}

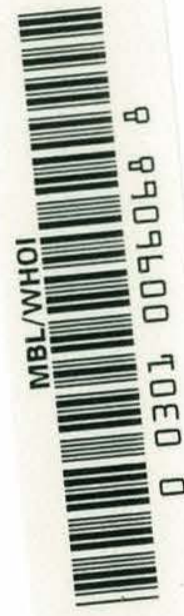
Funding was provided by the Office of Naval Research under contract Number N00014-86-K-0751; and by the National Aeronautic and Space Administration under contract No. 957652.
Reproduction in whole or in part is permitted for any purpose of the United States Government. This report should be cited as: Woods Hole Oceanog. Inst. Tech. Rept., WHOI-89-13.
Approved for publication; distribution unlimited.

\section{Approved for Distribution:}

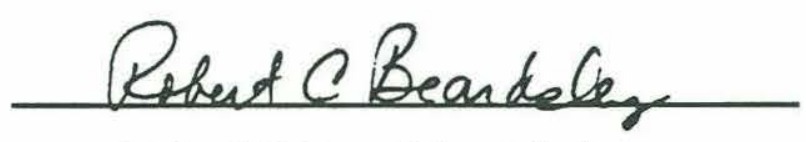

Robert C. Beardsley, Chairman Department of Physical Oceanography 



\section{Contents}

1 Introduction 1

2 System Requirements 1

3 Conventions 1

4 Window Organization 2

4.1 Menu Window . . . . . . . . . . . . . . . . . . . . 2

4.2 Display Window . . . . . . . . . . . . . . . . . . . . . 4

4.2 .1 Video Lookup Tables (VLTs) . . . . . . . . . . . . . . . 4

4.3 Cursor Window . . . . . . . . . . . . . . . . 5

5 Filetypes 5

5.1 ASCII Files . . . . . . . . . . . . . . . . . . 6

5.2 Binary Files . . . . . . . . . . . . . . . . . . . . . 6

5.3 Sun Rasterfiles . . . . . . . . . . . . . . . . . . . . 6

5.4 SDPS Format Files . . . . . . . . . . . . . . . . 6

6 Basic Image Display $\quad 7$

6.1 Window and Memory Numbering ... . . . . . . . . . . . 10

6.2 Using the Cursor Window . . . . . . . . . . . . . . . . . . 12

7 Basic Line Display 14

7.1 Window Scaling . . . . . . . . . . . . . . . . . 14

7.2 Converting data to SDPS format . . . . . . . . . . . 16

7.3 Lines . . . . . . . . . . . . . . . . . . . . . . . 17

7.3 .1 Additional line types . . . . . . . . . . . . 18

7.4 Vectors. . . . . . . . . . . . . . . . . . . . 18

8 Basic Image Enhancement 21

8.1 VLT Functions . . . . . . . . . . . . . . . . . . . . . . . . . 21

8.1 .1 Colorbar . . . . . . . . . . . . . . . . 23

8.1.2 Cursor Controlled Linear Mapping (CCLM) . . . . . . 25

8.2 Image Functions . . . . . . . . . . . . . . . . . . . 25

8.2 .1 Histogram . . . . . . . . . . . . . . . . . . . 25

8.2 .2 Zoom ................................ 25 
8.2 .3 Math . . . . . . . . . . . . . . . . . . . . . . . . 28

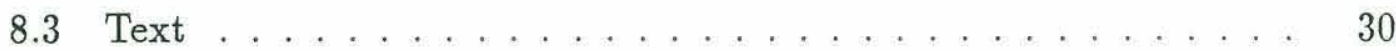

9 Basic Output 32

9.1 SDPS Format . . . . . . . . . . . . . . . . . . . 32

9.2 PostScript . . . . . . . . . . . . . . . . . . 33

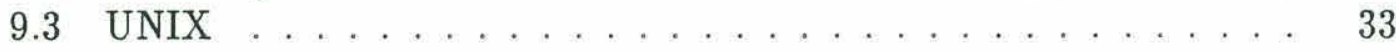

10 Advanced Functions 33

10.1 VLT Modification . . . . . . . . . . . . . . . 34

10.2 Movie Loop . . . . . . . . . . . . . . . . . . . . . . . . 34

10.3 Plotting Scalar Values Along a Track . . . . . . . . . . 36

10.4 Line Drawing . . . . . . . . . . . . . . . . . . . 38

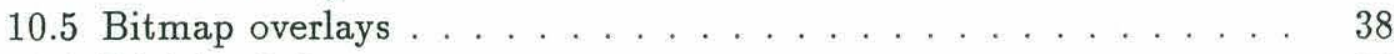

10.6 Digitize Points . . . . . . . . . . . . . . . . . . . . . . . . . . 39

10.7 Dithered Images . . . . . . . . . . . . . . . . . . . 39

10.8 Defaults . . . . . . . . . . . . . . . . . . . . . . . . 39

11 Menu Listings and Descriptions $\quad 39$

11.1 Window Functions . . . . . . . . . . . . . . . . . . . 39

11.2 VLT Manipulation . . . . . . . . . . . . . . . . . . . . . 41

11.3 Raster Image Display . . . . . . . . . . . . . . . . . . . . . 43

11.4 Track Data Plotting . . . . . . . . . . . . . . . . . . 44

11.5 File Reformatting . . . . . . . . . . . . . . . . 47

11.6 Data Processing Functions . . . . . . . . . . . . . . 47

11.7 Dither And PostScript Functions _. . . . . . . . . . . . 48

11.8 Image Functions . . . . . . . . . . . . . . . . . . . . . . . 48

12 External Utilities $\quad 49$

12.1 sdps_append . . . . . . . . . . . . . . . . . . . . . . . . 49

12.2 sdps_convert . . . . . . . . . . . . . . . . . . . . 50

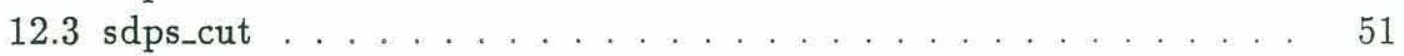

12.4 sdps_flip . . . . . . . . . . . . . . . . . . . 51

12.5 sdps_ftb . . . . . . . . . . . . . . . . . . . . . 52

12.6 sdps_header . . . . . . . . . . . . . . . . . . . . . . 53

12.7 sdps_headedit . . . . . . . . . . . . . . . . . . . 53

12.8 sdps $\_$logscale . . . . . . . . . . . . . . . . . . . . . . 53 
12.9 sdps_mask . . . . . . . . . . . . . . . . . 53

12.10 sdps_math . . . . . . . . . . . . . . . 54

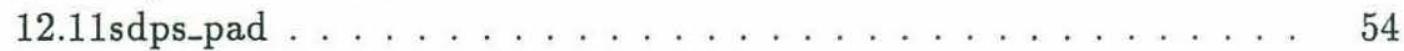

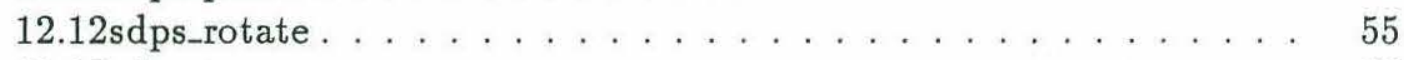

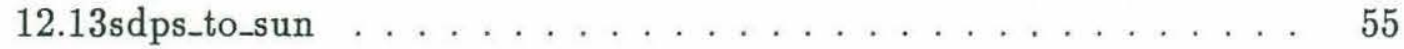

A Example ASCII Line data file $\quad 57$

B Example ASCII Vector data file $\quad 57$ 


\section{List of Figures}

1 SDPS with one open display window. . . . . . . . . . . . . 3

2 Example of an image printed on a PostScript laser printer. Image courtesy of NORDA. . . . . . . . . . . . . . . 11

3 Example of plotting a continuous line. Grid lines were added using option 42 and notation was added using option $46 \ldots . . . .19$

4 Example of a vector plot. Box was added using option 42 and notation was added using option 46. . . . . . . . . . 22

5 Example of a scalar plot. Coastline was added as a discontinuous

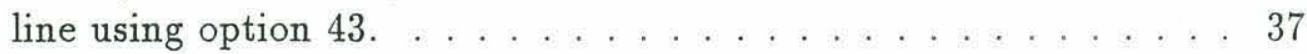




\section{Introduction}

SDPS is a menu driven interactive program designed to facilitate the display and output of image and line-based data sets common to telemetry, modeling and remote sensing. This program can be used to display up to four separate raster images and overlay line-based data such as coastlines, ship tracks and velocity vectors. The program uses multiple windows to communicate information with the user. At any given time, the program may have up to four image display windows as well as auxiliary windows containing information about each image displayed. SDPS is not a commercial program. It does not contain complete type checking or error diagnostics which may allow the program to crash. Known anomalies will be mentioned in the appropriate section as notes or cautions.

\section{System Requirements}

SDPS was designed to be used on Sun* Microsystems Workstations running SunView1 (Sun Visual/Integrated Environment for Workstations). It was primarily designed to be used on workstations equipped with color monitors, but most of the line-based functions and several of the raster-based functions can be used with monochrome monitors. The program currently runs on Sun3 series workstations running SunOS 4.0 and should port easily to Sun4 and Sun386 series workstations with SunView1. Users should also be familiar with UNIX ${ }^{\dagger}$, Sun workstations and the SunView window system.

\section{Conventions}

All window information is given in pixels except where the program requests degrees. Pixels are assumed to be integers while degrees are decimal degrees. File names are assumed to be complete with extensions. In the text the following conventions are used: .bin for unformatted binary; .sdps for SDPS type byte; .sdpsf for SDPS type float; and .asc for ASCII files ${ }^{1}$.

${ }^{*}$ Sun, Sun3, Sun4, Sun386 and SunView1 are trademarks of Sun Microsystems

${ }^{\dagger}$ UNIX is a trademark of AT\&T Bell Laboratories

${ }^{1}$ See section 5.4 for more information on file types 


\section{Window Organization}

SDPS is a windows-based program consisting of three basic windows (figure 1). The menu window used for input and output, the display window for lines and images and the cursor window for cursor information.

\subsection{Menu Window}

When SDPS is initiated by typing $\mathbf{s d p s}^{2}$, a menu window is opened showing the following main menu:

$\begin{array}{ll}\text { : SDPS - Satellite Data Proc. Sys. } & \text { : } \\ \text { : } 10 \text { Window functions } & : \\ : 20 \text { VLT Manipulation } \\ : 30 \text { Raster/vector image display } \\ : 40 \text { Track data plotting } \\ : 50 \text { File formatting } \\ : 60 \text { Data processing functions } \\ : 70 \text { Dithering functions } \\ : 80 \text { Image functions } \\ : 99 \text { Exit program } \\ \text { : Type <ESC> to abort function. } \\ :--\end{array}$

This window is used for text input and output by the user. This menu shows the list of available submenus. These additional menus are accessed by entering the appropriate number and pressing return. These commands will be described in subsequent sections. Note: if you type something incorrectly in this window, you can abort that function and return to the submenu by hitting the escape key $<$ ESC $>$. Also, typing a carriage return $\langle\mathbf{C R}>$ in any submenu will return you to the main menu. You can get to any function by simply typing the number of the function. You do not have to return to the main menu and enter each submenu. This is done in the basic examples to familiarize the reader with the program organization.

\footnotetext{
${ }^{2}$ See your system manager if you have problems starting the program with this command
} 


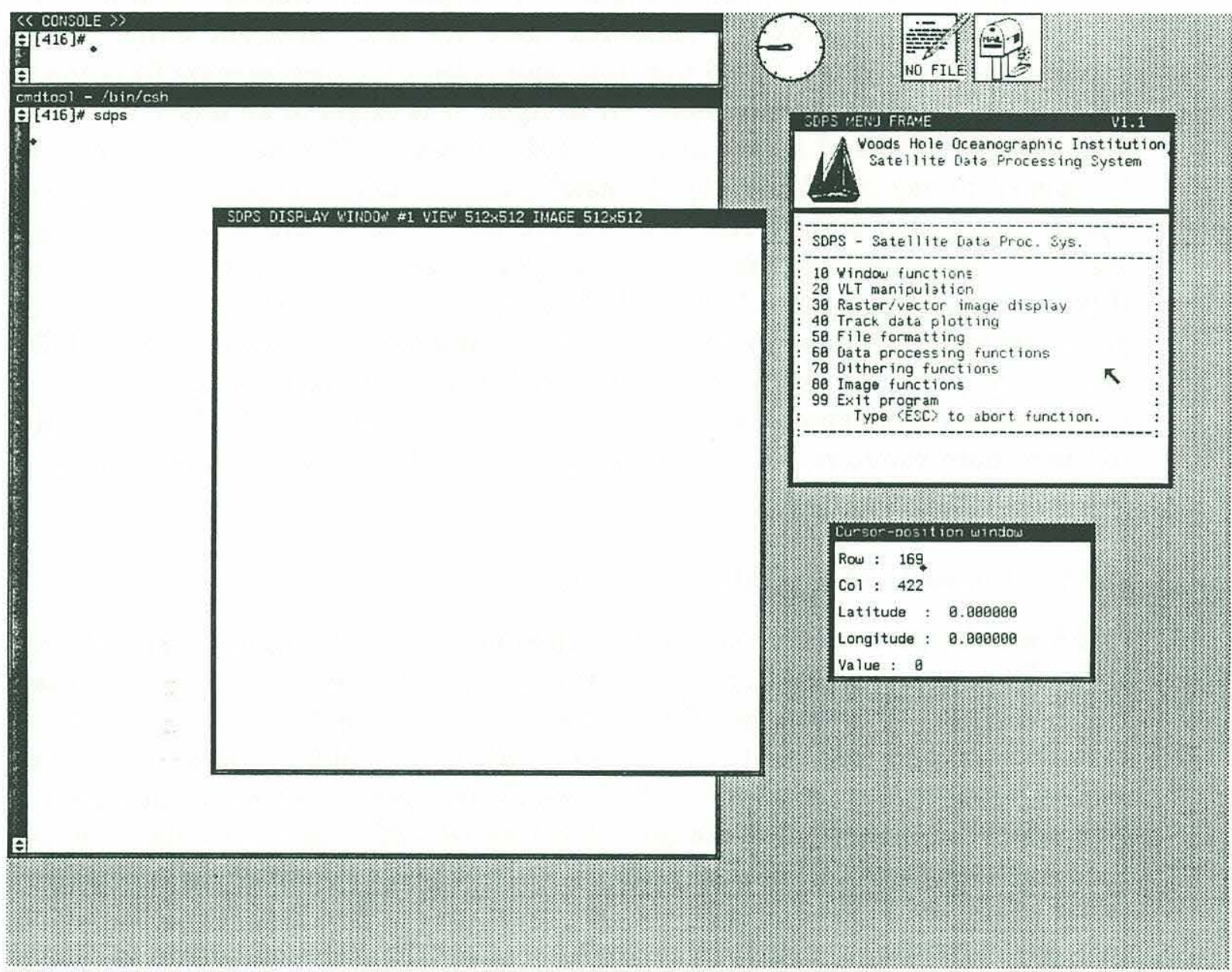

Figure 1: SDPS with one open display window. 


\subsection{Display Window}

This window is used for graphic input and output. All display windows start with the same configuration. Although there are many different terms for the coordinates of an image, SDPS uses rows and columns. Rows increase from top to bottom and columns increase from left to right. The origin of an image is located in the upper left hand corner at row 0 and column 0 . The display window may be opened to any size desired by the user ${ }^{3}$. If a window is larger than the screen size, the user may specify one value for the view window and a separate, larger value for the image size (window and image sizes are given in pixels). Therefore, if you wanted to display a 1536 by 1536 image on an 1152 by 900 Sun monitor, you could specify a view window of 700 by 700 and an image size of 1536 by 1536 . You would get a display window that was 700 by 700 pixels in size with scroll bars $^{4}$ on it. A maximum of four open display windows is allowed. If the user tries to open more windows, an error message is printed and the program returns to the main menu.

\subsubsection{Video Lookup Tables (VLTs)}

SDPS was designed to work primarily with 8-bit color workstations. This will only allow the program to display 256 individual colors at one time. However, there are more than 16 million possible colors to choose from to display. Therefore, a VLT is used to map up to 256 of the available 16 million colors. Each open display window may have its own VLT which becomes active when the cursor is placed in the window. Because all 256 colors are active in the display window, the foreground and background colors used in the text windows are temporarily switched out while the display colors are switched in. This may be disconcerting since the only window visible is the display window. Information on how to change this effect will be given later. When using the program with a color or greyscale monitor, each display window is opened and given a linear greyscale VLT. The colors in this map run from pure black to pure white.

\footnotetext{
${ }^{3}$ Practical limits vary from 2048 by 2048 to 4096 by 4096 depending on system configuration

${ }^{4}$ See Sunview Beginners Guide
} 


\subsection{Cursor Window}

This window is also opened when the program starts and should not be removed. The window may be moved or closed if the user desires.

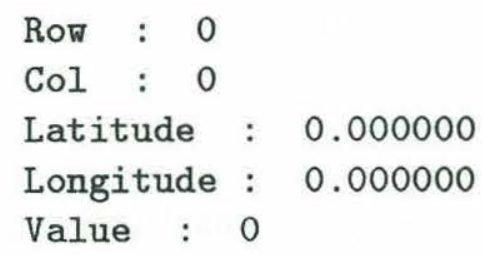

This window is used to display continuous information to the user. When the cursor enters a display window, it changes to a cross hair and the cursor window gives information specific to that window. The row and column numbers are always updated as is the value number (the original pixel value) under the cursor. The latitude and longitude only change after window specific navigation information has been entered. More detailed information is given in section 6.2.

\section{$5 \quad$ Filetypes}

Data files to be displayed may be of two distinct file types: image (or rasterbased) and vector (or line-based). An image file is a two dimensional function $\mathcal{F}(x, y)$. When displaying an image visually, this function is represented by the light intensity displayed. A digital image is the result of spatially discretizing the image function $\mathcal{F}$. SDPS currently only supports digital images. All unevenly or irregularly spaced images must be discretized to an even spatial grid. Each dimension may have a different spacing, but it must be constant for that direction.

The line data type contains a series of one or more points. Lines may be continuous, discontinuous, discrete points or vectors. Both data types may be composed of either byte values, integer values or floating point values. SDPS was primarily designed to read and write a specific file format (section 5.4). This format can handle both image and line data types and is in binary format to make reading and writing faster. All data must be converted to SDPS format before using SDPS. The only exceptions to this are Sun rasterfiles (section 5.3) and simple binary images (section 5.2). The idea behind this is that it is better to have a standard format for reading and manipulating images than it is to continually modify code to accept new formats. The SDPS format is given for users who 
would like to write conversion programs (section 5.4). Binary images may be read in to allow the user to preview an image before doing any format conversion. Sun rasterfiles may be read in to maintain system compatibility. Note: all images must be converted to byte images before using SDPS to display them. However, many external routines (section 12) support additional image types.

\subsection{ASCII Files}

ASCII files are not directly supported by SDPS due to speed considerations. The program does include one option (option 51, section 11.5) as well as an external routine sdps_convert (section 12.2) to convert ASCII files. It is recommended that the latter be used since it is a more robust routine.

\subsection{Binary Files}

The only non-SDPS format binary files supported are byte-encoded digital images. These images may have any size header, but any information contained in them is ignored. Binary files may also be converted to SDPS format files using option 52 (section 11.5).

\subsection{Sun Rasterfiles}

The Sun rasterfile format is the basic format for image files used by many Sun workstation programs. The rasterfile is composed of three parts. The first part is the 32 byte header. This header contains 8 integers that described various attributes of the image such as the number of rows, the number of columns and the number of bits per pixel in the image. The second part contains the colormap values. The size of this section is variable from image to image and is given in the header. The third part is the actual image stored row by row. For more information refer to the rasterfile manual page in the file formats section of the Sun manuals.

\subsection{SDPS Format Files}

Most options to SDPS require that input data files be in SDPS format. This is needed since the program requires information when reading the file and often when manipulating the data in memory. This information includes the size, the 
type of data, etc. An SDPS format file is simply a binary file with a 512 byte header. The header format is :

\begin{tabular}{|c|c|c|c|}
\hline \multicolumn{4}{|c|}{ SDPS Header Format } \\
\hline Variable & Type & Bytes & Description \\
\hline annot & char & 80 & $\begin{array}{l}80 \text { character array used for identifying the } \\
\text { image }\end{array}$ \\
\hline type & long int & 4 & $\begin{array}{l}\text { type of data: } \\
1 \text { - Byte }\left(\text { Logical }^{*} 1\right) \\
2 \text { - Short integer (Integer*2) } \\
3 \text { - Long integer (Integer*4) } \\
4 \text { - Float (Real*4) } \\
5 \text { - Complex (Complex) }\end{array}$ \\
\hline $\operatorname{dim}$ & long int & 4 & number of dimensions in data file $(\max 4)$ \\
\hline $\operatorname{ind}[4]$ & long int & 16 & number of indices for each dimension \\
\hline inc [4] & float & 16 & increment between indices in each dimension \\
\hline slope & float & 4 & $\begin{array}{l}\text { slope of linear relationship between byte and } \\
\text { float data }\end{array}$ \\
\hline intrcp & float & 4 & $\begin{array}{l}\text { intercept of linear relationship between byte } \\
\text { and float data }\end{array}$ \\
\hline unused[384] & char & 384 & $\begin{array}{l}\text { fills } 512 \text { byte record for data exchange with } \\
\text { FORTRAN programs }\end{array}$ \\
\hline
\end{tabular}

The data segment of an SDPS format file may be any of the five types listed above, but the two most common formats are byte and float. The other types are included for future expandability. SDPS can read both byte and float files, however image functions currently only support byte type files and the line functions only support float type files. The are several external programs (section 12) that support both types of files.

\section{Basic Image Display}

To display an image using SDPS, the user must know the format and size of the image to be displayed. The format must be either binary, Sun rasterfile or SDPS as described in section (5). The image size must be known in order to open the correct size window. The image size as well as the header size of binary files must 
be obtained by the user. The image size for Sun rasterfiles can be obtained from the UNIX command 'file'. The image size for SDPS format files may be determined using the external routine sdps_header (section 12.6). Assuming the file is a binary format file 1024 rows and 768 columns and has a 512 byte header, the first step is to open a window using option 11. Program output is in Typewriter, user input is in Boldface, comments are in Italics:

: SDPS - Satellite Data Proc. Sys.
$:$ :
$: 10$ Window functions
$: 20$ VLT Manipulation
$: 30$ Raster/vector image display
$: 40$ Track data plotting
$: 50$ File formatting
$: 60$ Data processing functions
$:$ :
$: 80$ Dithering functions
$: 99$ Exit program
$:$ Type <ESC> to abort function.
$:-1$

10

Select the Window Functions Submenu

: SDPS - Satellite Data Proc. Sys.
$: 11$ Open window
$: 12$ Redisplay image
$: 13$ Flick between images
$: 14$ Output window to memory
$: 15$ Output window to file
$: 16$ Output B+W remapped window to file :
$: 17$ Clear window
$: 18$ Display colorbar
$: 19$ Dump window to unix plot file
$:$ Type <ESC> to abort function.
$:-1$


Enter size of view window \#1 (row, col)

Default: [512 512]

768768

Select a window size in pixels that will fit on the screen

Enter full image size (row, col): [512 512]

$1024768 \quad$ Enter the actual size of the image in pixels

A display window will be opened up on the screen 768 pixels high by 768 pixels wide. Also the cursor window will be opened up when you open the first display window. Next load the image into the display window:

: SDPS - Satellite Data Proc. Sys.

: 31 Display Sun rasterfile

: 32 Display binary image file

: 33 Display SDPS image file

: 34 Display multiple SDPS image files :

: 35 Display SDPS mask file

: 36 Overlay SDPS bit map

:

:

:

: Type $\langle E S C\rangle$ to abort function. :

Enter filename of input image ?

file1.bin Enter the filename of the image

Enter image memory \# and window \# [1 1 ] 
Enter display position (row, col) [0 0]

$00 \quad$ Position it in the upper left hand corner

Enter image size (row,col) [1024 768]

$1024768 \quad$ Enter the image size

Enter \# of bytes of header to skip [0]

512

Enter the size of the header to skip

The image should be loaded into the window with a black and white VLT. The menu will return to the Raster/image display submenu. When the cursor enters the display window, the black and white VLT will become active and the image will become visible. The scroll bars can now be used to view the hidden sections of the image. Note: most of the scroll bar functions have not been implemented. To move to a different part of the image, the user must use the middle mouse button to move to the section of the image relative to the position of the cursor in the scroll bar. An example of this type of image is given in figure 2. This image was created by NORDA and was generated using option 74. For more information on creating hardcopy of images see section 9. Note: User entries are separated by spaces not commas.

\subsection{Window and Memory Numbering}

SDPS supports up to 4 concurrent display windows and up to 50 virtual windows 5 . A virtual window is a copy of a displayable image or window stored in virtual memory and not as disk file. Auxiliary information such as colormaps are not stored along with an image in memory. Therefore memory locations should be used when this information is not needed. For example, the user may want to recall an image of a coastline and not be concerned with knowing the pixel values. The coastline can be quickly re-displayed from memory rather than being read from disk again.

\footnotetext{
${ }^{5}$ Actual amount depends on image sizes and system configuration.
} 


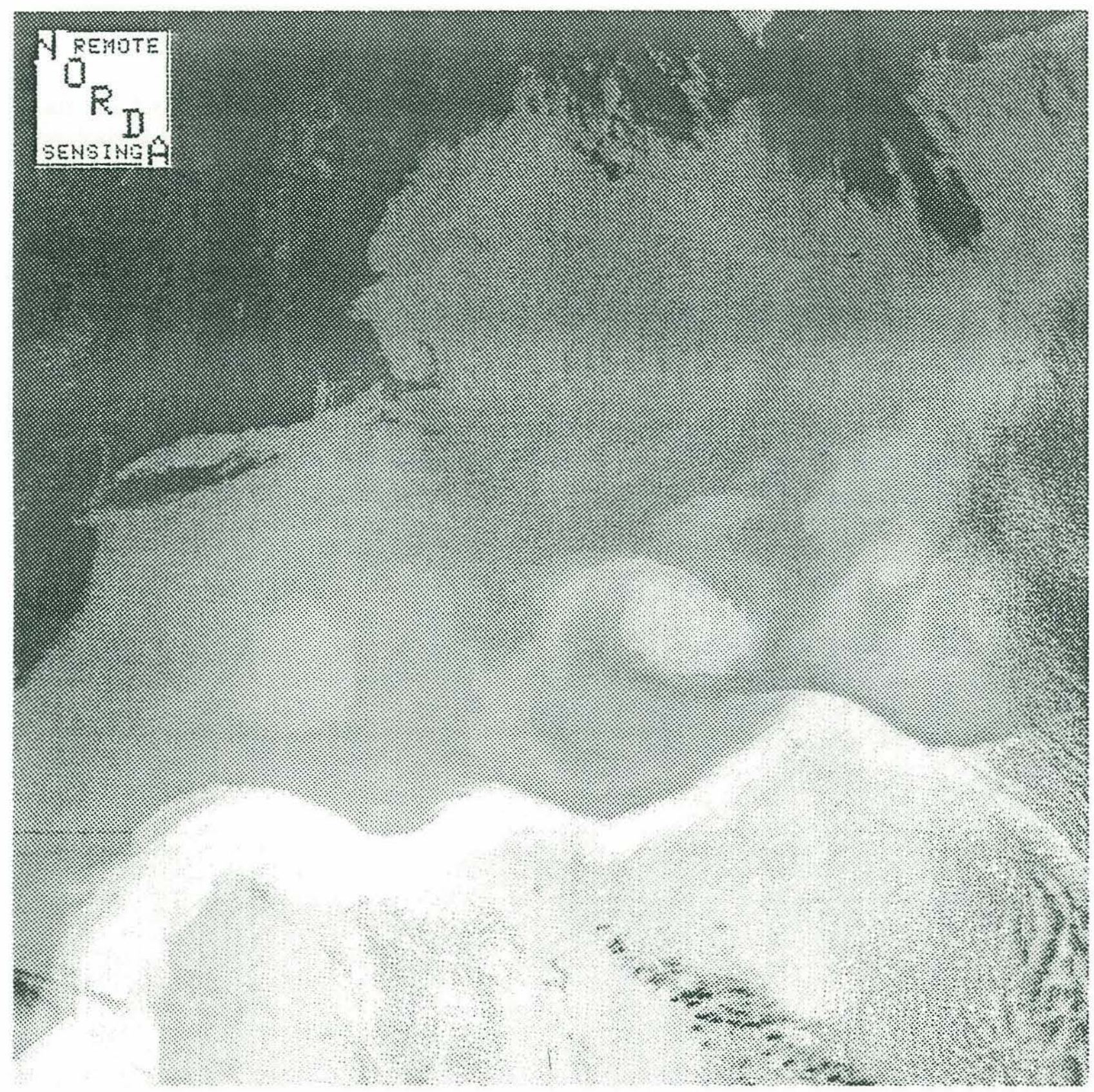

Figure 2: Example of an image printed on a PostScript laser printer. Image courtesy of NORDA. 
Each time an image display window is opened, it is numbered from 1 to 4 and its number is shown in the title bar across the top of the window unless the window is too small to display it. If the user opens two windows and then quits window number one, the next window opened will be window number one. A window may be eliminated by selecting the quit option from the window menu.

\subsection{Using the Cursor Window}

The cursor window is opened when a display window is opened. This window gives information about cursor position and pixel values. Since SDPS uses 256 greyshades by default (section 4.2.1), the cursor window is not visible when the cursor is in the display window. To make the cursor window visible, the user must truncate the VLT of the display window. This can be done as follows:

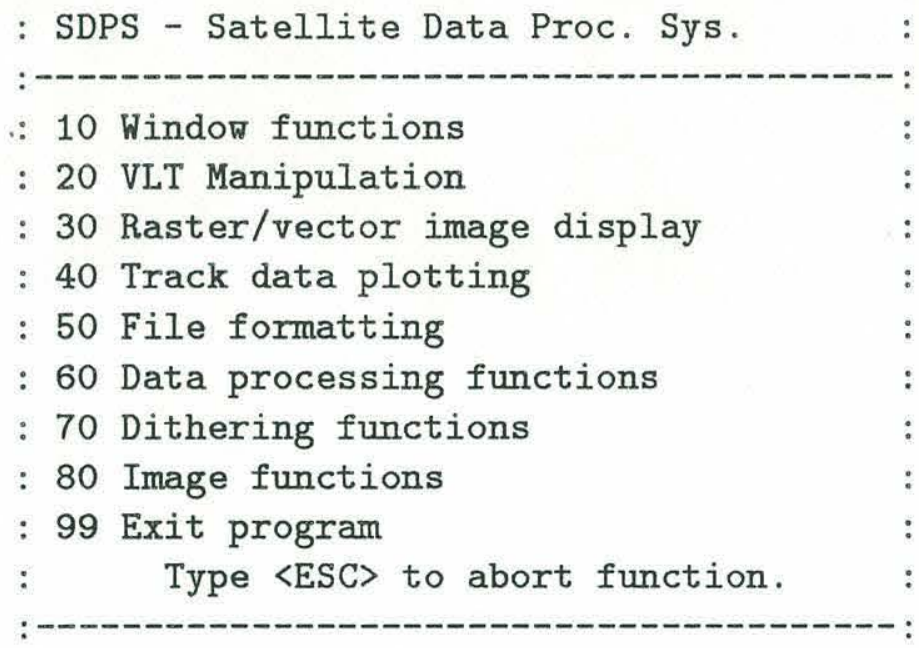




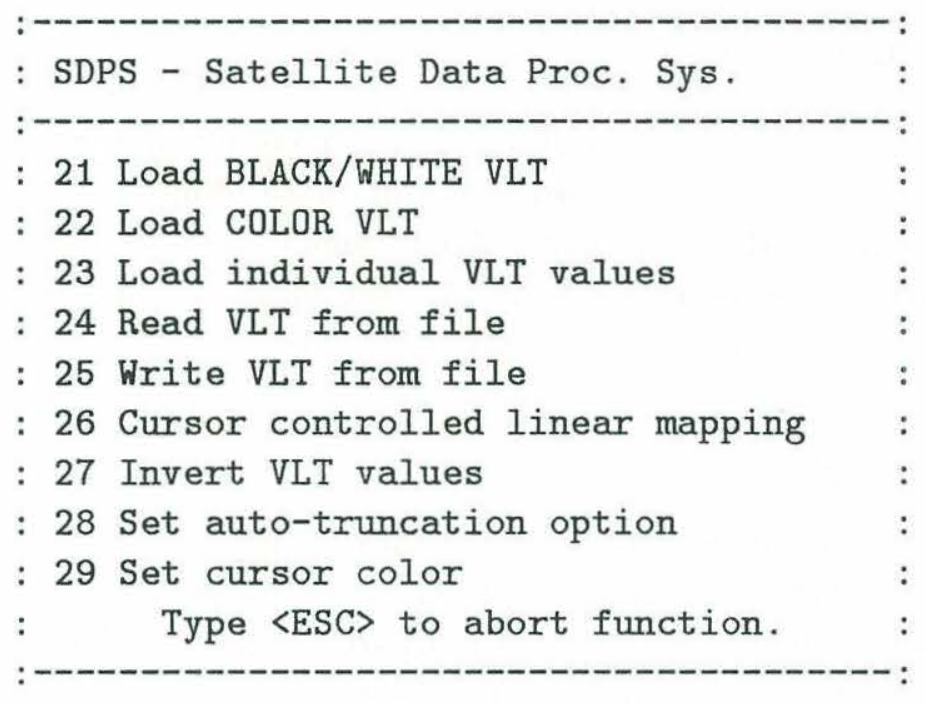

28 Select the auto-truncation option

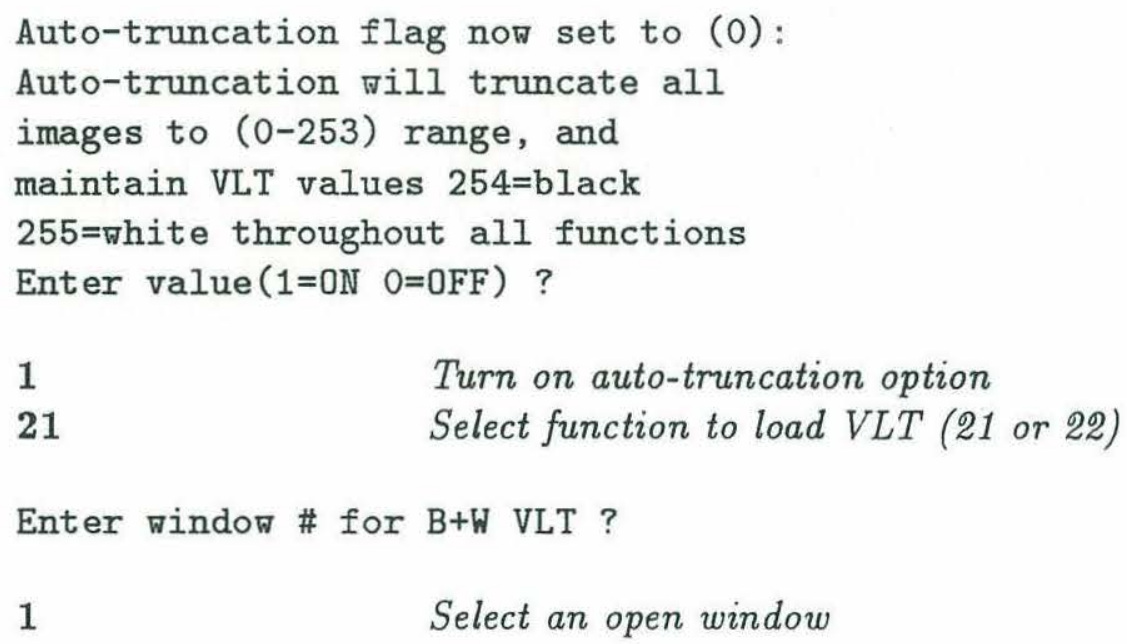

Now when the cursor enters the display window, the cursor location will be displayed along with the value of the pixel under the cursor. Activating the latitude/longitude display is demonstrated in section 7.1. 


\section{Basic Line Display}

Often it is useful to display various types of lines such as coastlines, wind vectors, ship tracks, satellite ground tracks or buoy information on an image. This section will describe how to accomplish this. Appendix A contains a data set that will be used in the examples given in sections 7.1 and 7.3. Appendix B contains a data set that will be used in the example given in sections 7.4 and 7.2.

\subsection{Window Scaling}

Before any line or vector drawing can be done, the window must be scaled. Since this program was designed for oceanographic data, the windows may be scaled using 3 projections: Stereographic, Mercator and Equirectangular. Although all projections are based on earth coordinates, the equirectangular projection can be given almost any linear coordinates. An example of setting the projection to equirectangular for a flat earth type of projection is shown. The data in appendix A will fit entirely in a latitude/longitude box $25 \mathrm{~N}, 285 \mathrm{E}$ to $35 \mathrm{~N}, 295 \mathrm{E}$. First open a window as described in section 6 with a view size of 512 by 512 and an image size of 512 by 512 . This function will work with any size window, however the area of interest is square and 512 by 512 is a convenient screen size. Note: longitudes should generally be given in east coordinates. West coordinates will work with most functions, but are not fully supported. The user should be cautious when the window spans 0 degrees longitude whether using east or west coordinates. 

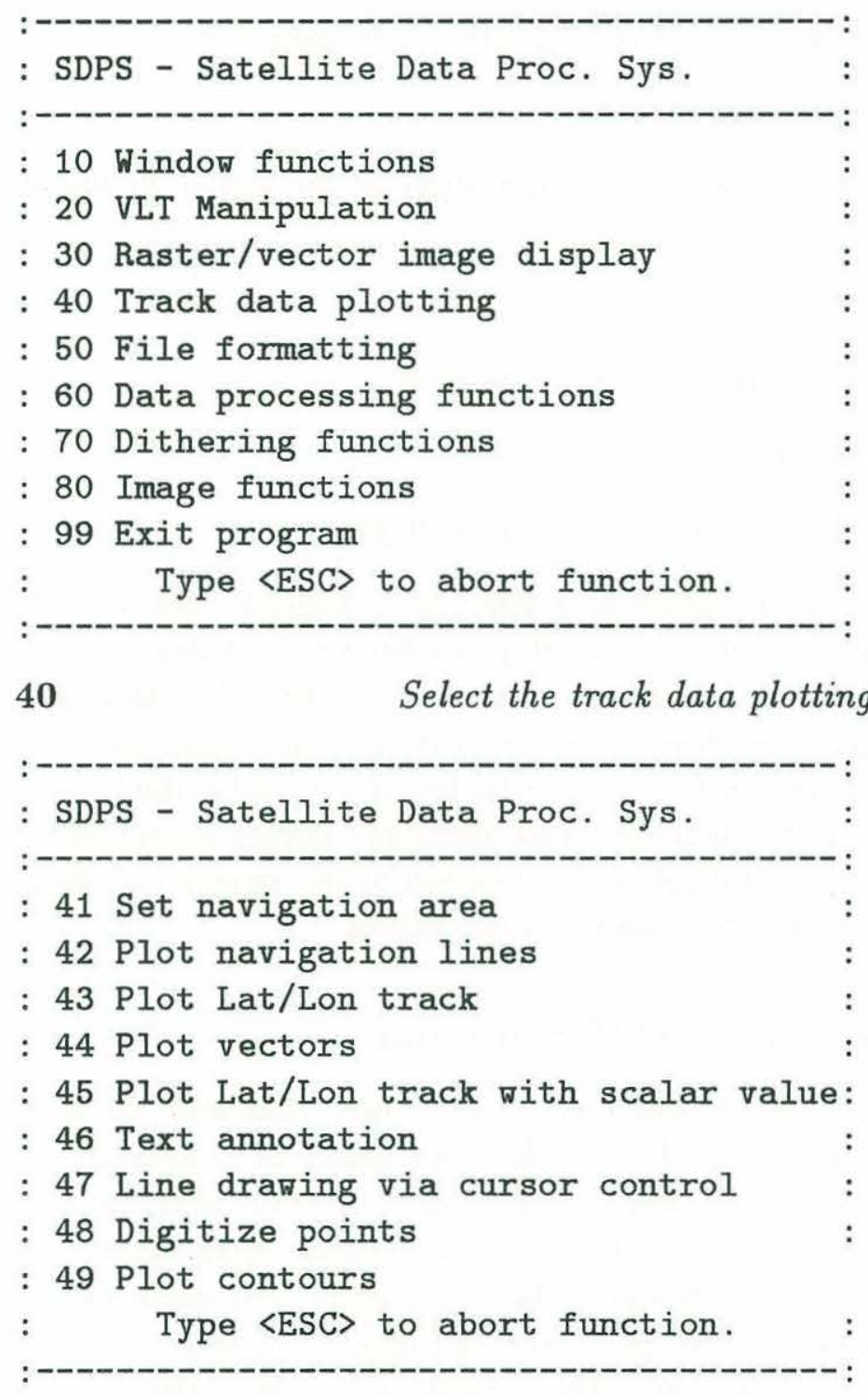

41

Select the set navigation area function

Enter navigation type:

1) Stereographic

2) Mercator

3) Equirectangular

Enter choice? 
Enter center latitude, longitude ?

30290

Set the latitude and longitude at the center of the window

Enter rotation angle?

0

Set the rotation angle to zero

Enter enter $\mathrm{x}$, $\mathrm{y}$ scale factor ?

2424

Set the column and row scale factors

Scaling factors are chosen with respect to a 360 degree window. An x scale factor of 1 would give a window scaled $+/-180$ degrees from the center longitude. A scale factor of 2 would scale the window $+/-90$ degrees etc. The same is true for the latitude or $y$ direction regardless of the window size. If a window is twice as wide as it is high and the user enters a scale factor of 11 , both the latitude and longitude will be scaled to 360 degrees. Now when the cursor enters the display window, the cursor window will display the location of the cursor in latitude and longitude coordinates as well as row and column values.

\subsection{Converting data to SDPS format}

To make file input faster, SDPS will only read binary files and all non-image files must be in SDPS format. ASCII data files can easily be converted to SDPS format. The ASCII data can be in any format and each point must be separated by a space or a tab. There should not be any extraneous data such as headers or labels and sequential data points should be in columns. For example, the data set in appendix B could be used to plot vectors (option 44, section 11.4) where the first column contains the longitude, the second column the latitude, the third column the $u$ velocity and the fourth column the $v$ velocity. To convert this to SDPS format the user should use the external function sdps_convert (section 12.2):

sdps_convert $224<$ file1.asc > file1.sdpsf

The number 22 is the number of rows in the input file and 4 is the number of columns. The user can now display lines and vectors as shown in the following sections. 


\subsection{Lines}

This data file can now be plotted in the display window. Assuming that the scaling has been set as in section 7.1 and the user wants to plot a line connecting the positions of each point (e.g. a ship track):

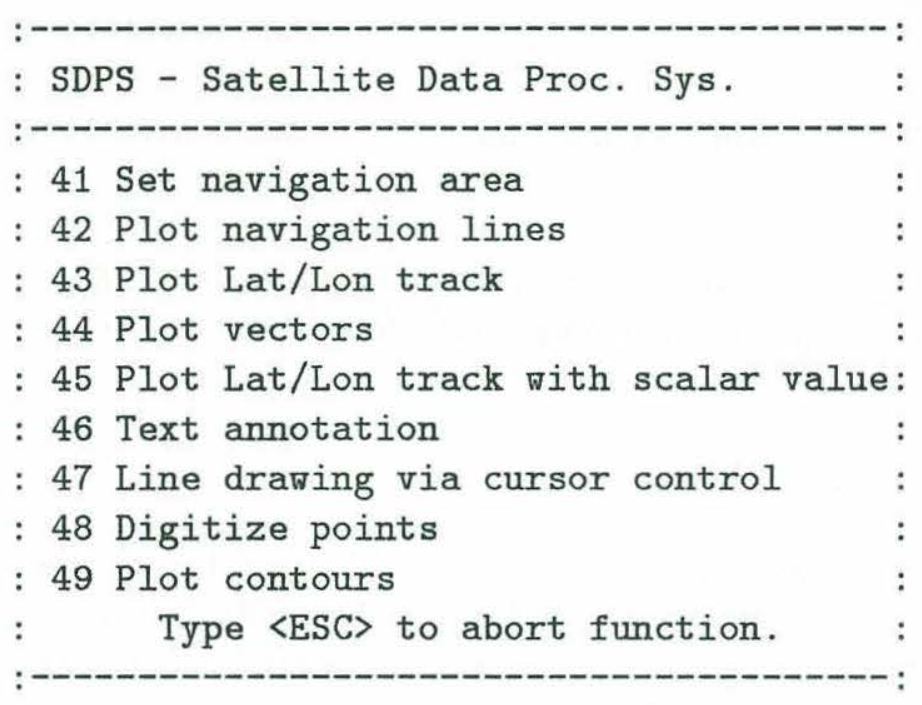

Enter window \# to use ?

Enter SDPS input file name ?

file1.sdpsf Enter the filename to read

Enter indices of lat, lon?

21

Column 2 contains the latitude and column 1 contains the longitude

Enter value to use $(0-255)$ ?

255

Enter the VLT value to use in plotting (white) 


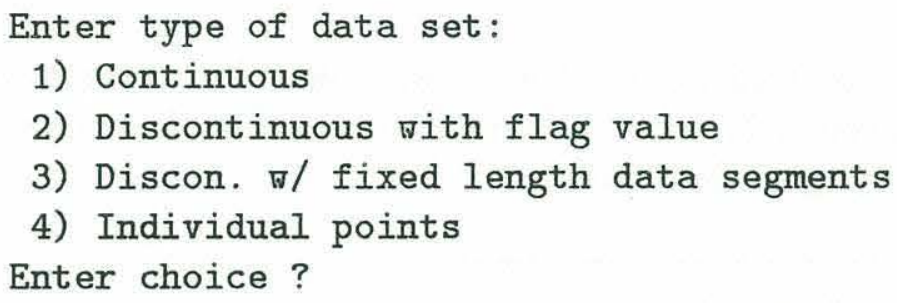

1) Continuous

2) Discontinuous with flag value

3) Discon. $/ /$ fixed length data segments

4) Individual points

Enter choice ?

Plot a continuous data set

A line connecting the data points will now be drawn in the window (figure 3 ). If the window had an image displayed, the line would have been drawn on top of the image.

\subsubsection{Additional line types}

SDPS will also read three other types of line data. The most commonly used option is Discontinuous with flag value. The data set has a flag such as -9999.0 -9999.0 for the latitude/longitude pair separating continuous line segments. The user can also plot individual points. A plus sign is plotted at each coordinate in the data file. Finally, the Discon. $w /$ fixed length data segments option plots multiple line segments each containing the same number of points.

\subsection{Vectors}

The data file in appendix A can also be used to plot vectors in a display window. Since the data will fit in the same size latitude/longitude box, the scaling should be set the same as in section 7.1. This example will display the data set as vectors with the length proportional to the magnitude (e.g. wind vectors): 


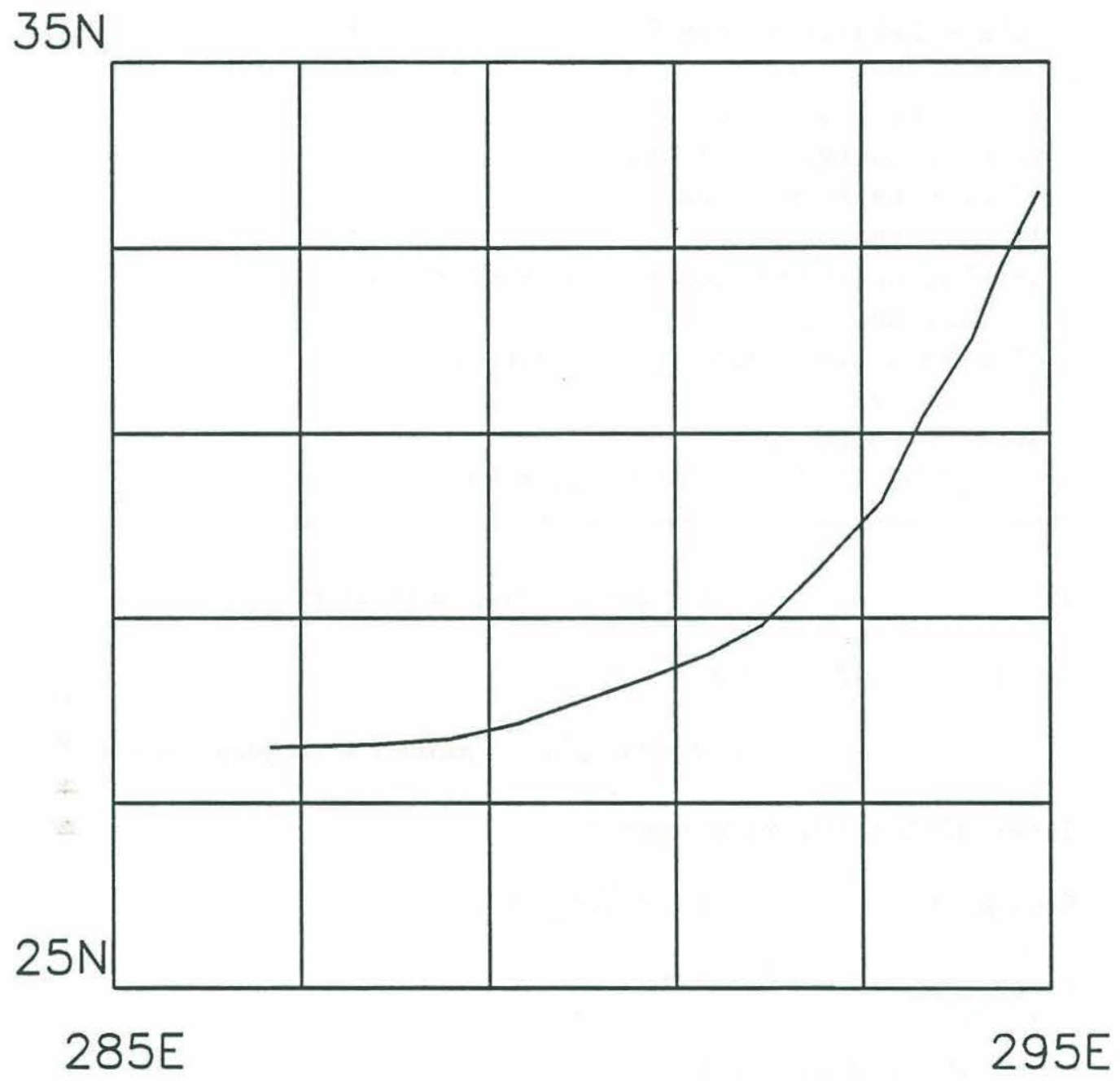

Figure 3: Example of plotting a continuous line. Grid lines were added using option 42 and notation was added using option 46 


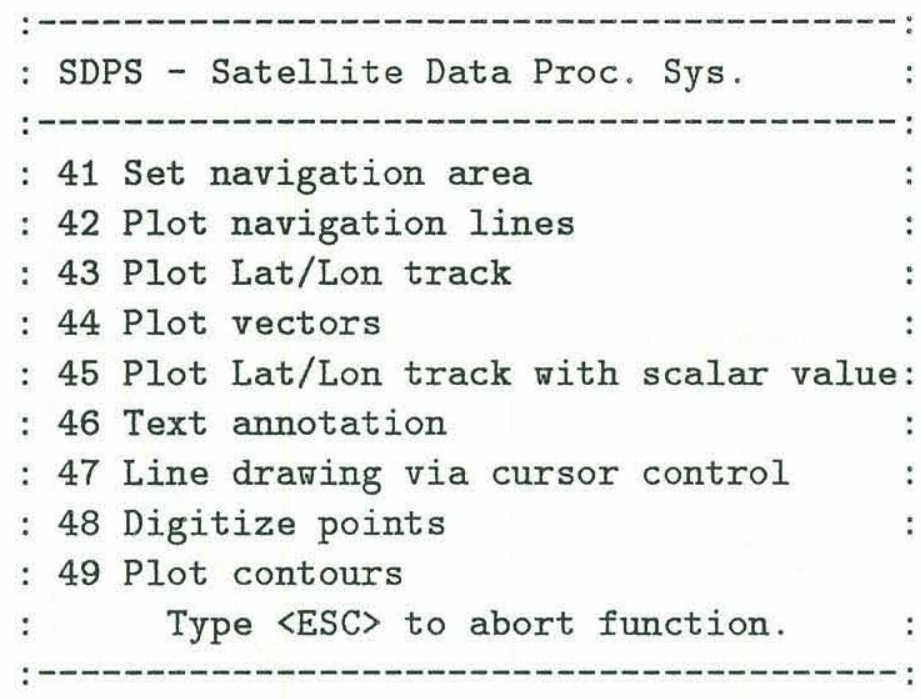

44 Select the plot vectors function

Enter window \# to use ?

Enter the window number to display vectors

Enter SDPS input file name ?

file1.sdps Set the filename to read

Enter type of vector data:

1) $U, V$

2) Magnitude, Direction

Enter choice?

1

Select $U, V$ input data type

Enter indices of Lat, Lon, U, V ?

2134 Column 2- lat, column 1 - lon, column 3-U, column 4-V

Enter max expected wind speed ? 
Enter type of plot:

1) Vector length varies with magnitude

2) Vector value varies with magnitude

Enter choice?

1

Select varying vector length option

Enter length of $\max$

vector in degrees of longitude?

2.0 A vector with a value of 7.0 will be have a length the same number of pixels as two degrees of longitude

Enter value to use $(0-255)$ ?

Arrows showing the magnitude and direction will now appear in the display window (figure 4). If the display window had an image, the arrows would be drawn on the image. If the type of plot selected is 2 , all vectors would be the same length and the color would specify the magnitude.

\section{Basic Image Enhancement}

This section will explain how to modify a display window and to obtain useful information about the data in the window. The user can modify the VLT, view a magnified section of a display, combine information from two windows or add colorbars and text to the display.

\subsection{VLT Functions}

SDPS defaults to a greyscale VLT when the user opens a new window. A user may want to display the current values or to change the VLT to display false colors or to change the mapping of the VLT to the display values. 


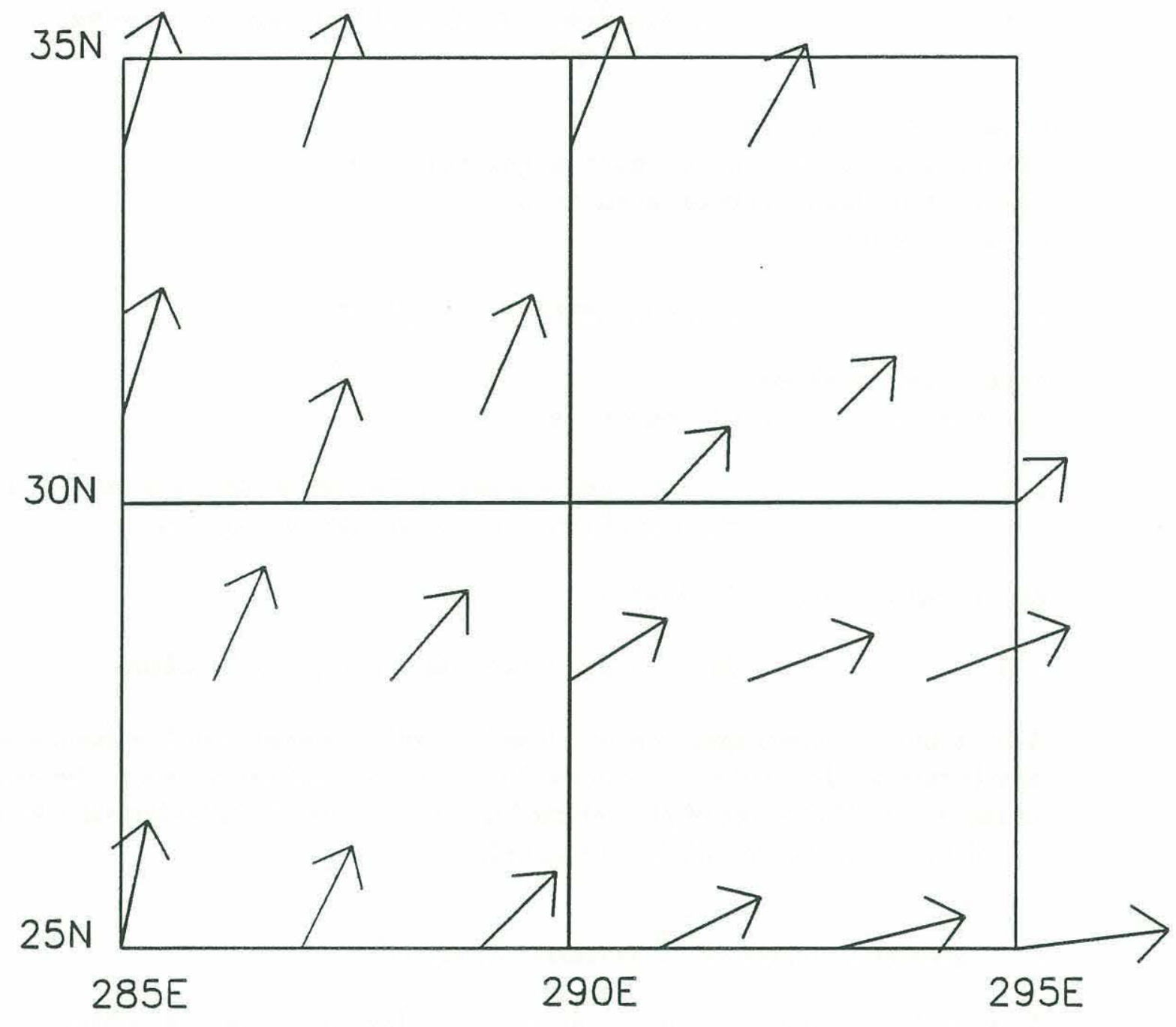

Figure 4: Example of a vector plot. Box was added using option 42 and notation was added using option 46. 


\subsubsection{Colorbar}

The current VLT may be displayed in a colorbar in a separate window, or it may be placed in a display window over an image. Assuming the user has opened a window and loaded an image as described above, a colorbar can be overlaid on an image with the following steps:

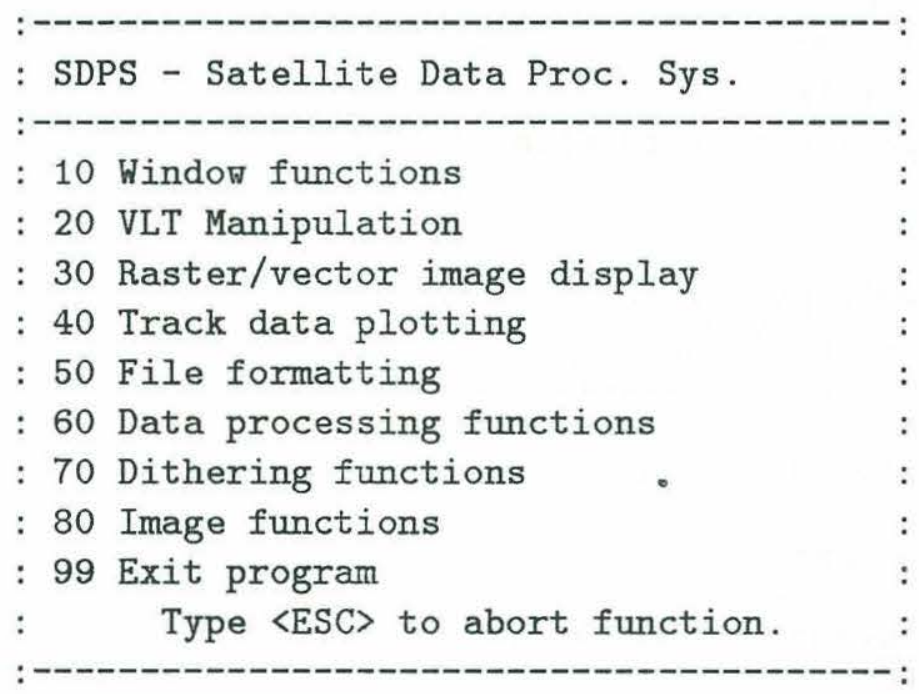




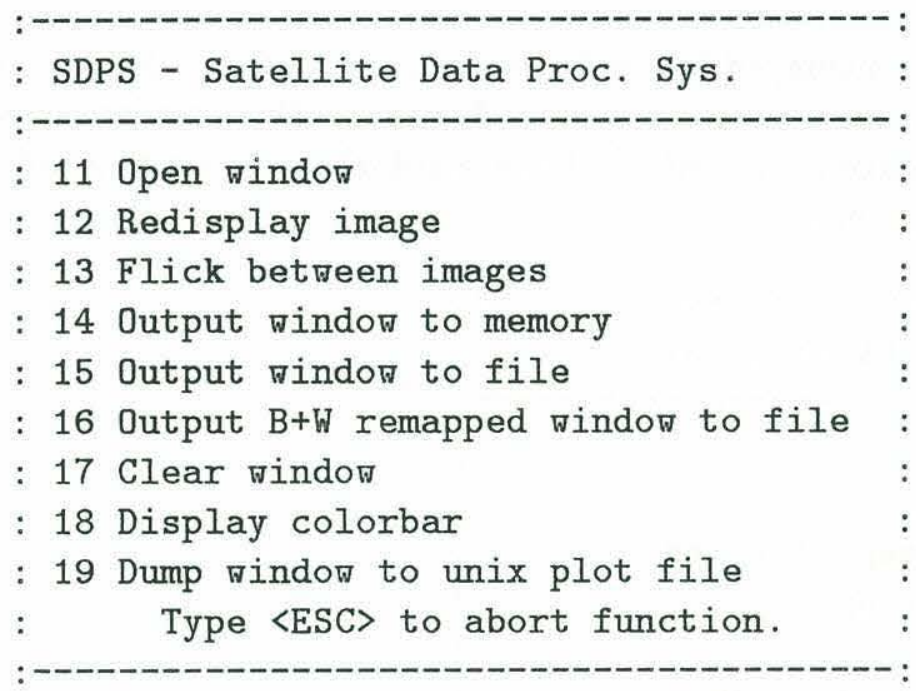

$18 \quad$ Select Display colorbar function

Enter window \# for colorbar ?

$1 \quad$ Enter the window number

Place colorbar (0)inside or (1)outside

0 Specify that the colorbar be placed inside the window

Enter row, col, height, width:

$101520256 \quad$ Enter the starting location, height and width in pixels

This will put a colorbar in the display window 10 rows from the top and 15 columns from the left. The colorbar will be 20 pixels high and 256 pixels wide. The colorbar can also be put into a separate window by selecting option 18 . Enter the window number to read the VLT and enter 1 to specify that the colorbar be placed outside the window. A window in the upper left hand corner of the screen will be created. If the image loaded was an SDPS format file (i.e., if the header contains information on pixel values, section 5.4), the values corresponding to the different colors will be displayed. Note: when placing a colorbar inside a window, if the height is greater than the width, a vertical colorbar will be drawn. Otherwise, a horizontal colorbar is drawn. Also colorbars inside windows must be annotated by hand. 


\subsubsection{Cursor Controlled Linear Mapping (CCLM)}

The easiest way to modify the VLT of a window is to use the CCLM option. When

this option is selected, the the cursor position controls the VLT map. Moving the cursor vertically changes the contrast; moving it horizontally changes the brightness (black and white) or the color range. To change the VLT, move the cursor and press the left mouse button. The original VLT can be recovered by pressing the cursor one quarter from the top in the middle of the window, or by reloading a VLT (options 21 and 22). The CCLM function is chosen by selecting option 26 and entering the window number to use. Now when the left mouse button is pressed in the window selected, the VLT will be remapped accordingly. The new VLT may be saved using option 25 and restored later using option 24 .

\subsection{Image Functions}

SDPS also provides functions for extracting additional information about images. There are functions to display a histogram of the image, magnify a section of a display window, perform simple math operations on two windows and overlay text.

\subsubsection{Histogram}

The histogram function reads the pixel values from a specified window and graphically displays the results in a new window. The window shows the number of pixels of each possible value (0-255) on a logarithmic scale. The colors in the histogram correspond to the colors in the display window from which the histogram was computed. A histogram is generated by selecting option 83 and specifying the window number.

\subsubsection{Zoom}

SDPS also provides a mechanism for magnifying an image (panning is automatically provided with the use of scroll bars). There are two types of magnification available. The user may select a simple pixel replication or a bi-linear interpolation. The replication method is much faster and allows the user to stretch an image by magnifying more in one direction than the other. The interpolation method is slower and smooths edges in the process. Interpolating sharp features such as lines and vectors may produce undesirable effects. Assuming the user has 
loaded an image as described above (section 6 ), the following example shows how to magnify a section of the image.

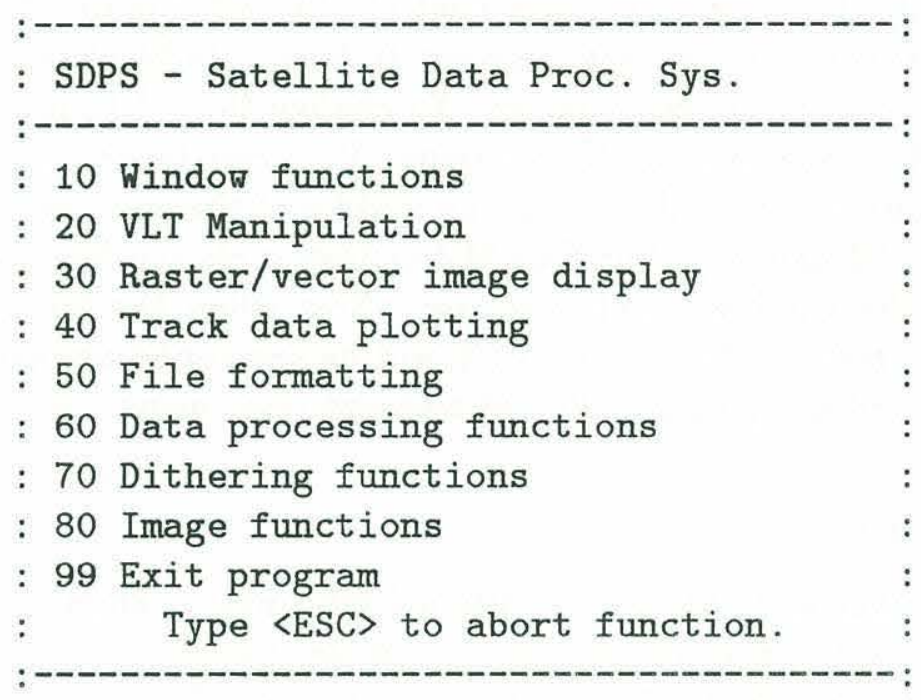

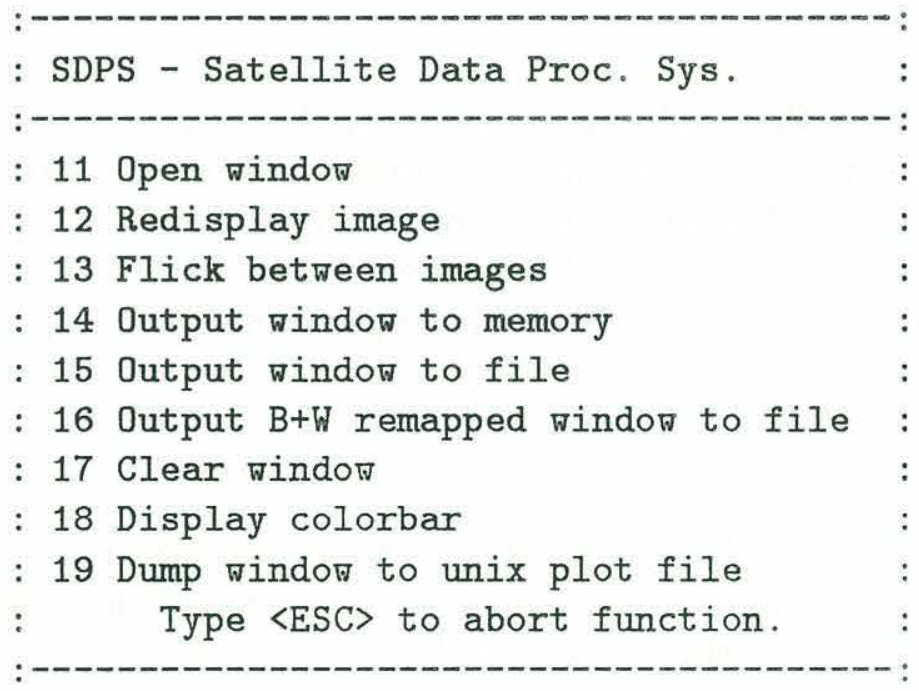

Enter size of view window \#2 (row, col) Default: [512 512] 
384384

Select a window size for the magnified image

Enter full image size (row, col): [512 512]

384384

Select a view size that will hold the magnified image

80

Select the Image functions submenu

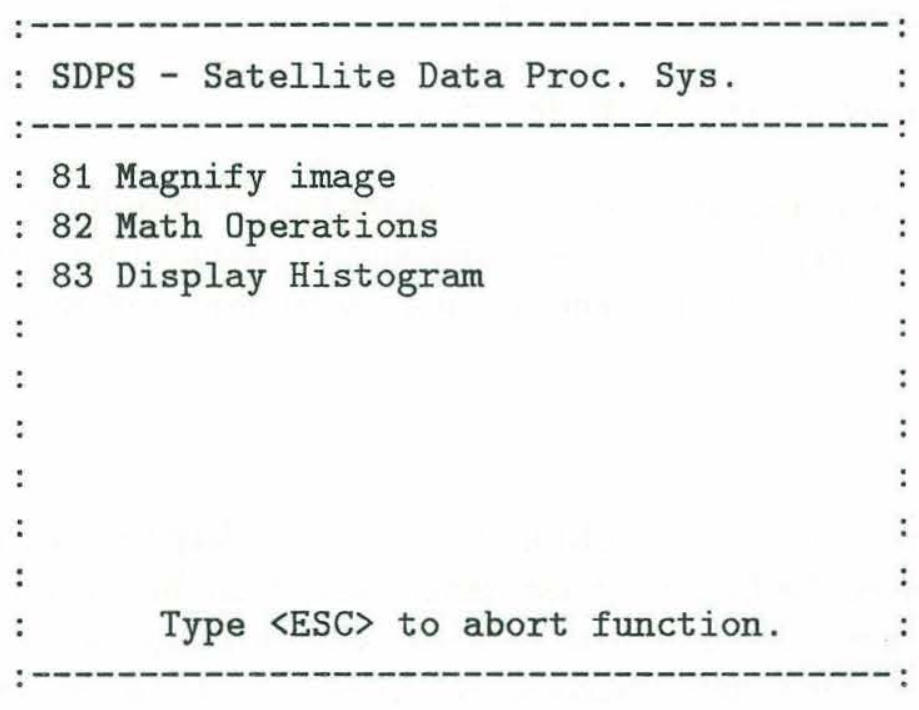

81 Select the Magnify image function

Enter window \# to read from ?

1

Select the window to read from

Enter memory \#, window \# to write to ?

$22 \quad$ Select the memory number and window to put the magnified image

Enter magnification type?

1 - Pixel Replication

2 - Bi-linear Interpolation

1 Select simple pixel replication

Enter magnification factors(row, col) ? 
Enter window upper left corner (row, col)?

00

Select the upper left corner

Enter window lower right corner (row, col)?

9595

Select an area 96 by 96 pixels

The magnified image will now appear in window number two. The bi-linear interpolation method linearly interpolates between rows and columns to magnify the image. The magnification factor is the same for both directions and must be a power of two.

\subsubsection{Math}

SDPS allows the user to add, subtract, multiply or divide one displayed image by another. These images must be SDPS format images and must be loaded using option 33. The images are first converted back to floating point values using the slope and intercept header values described in section 5.4. Then the selected option is performed and the result transformed back to byte values. The user may choose to keep the original byte mapping or have the program calculate a new slope and intercept. In all operations, the first image is operated on by the second and replaces the second, i.e., image2 $=$ image1 $\left[+_{-}^{*} /\right]$ image2. Assuming the user has two windows open and wants to find the difference between the two images, the following steps would accomplish that. 


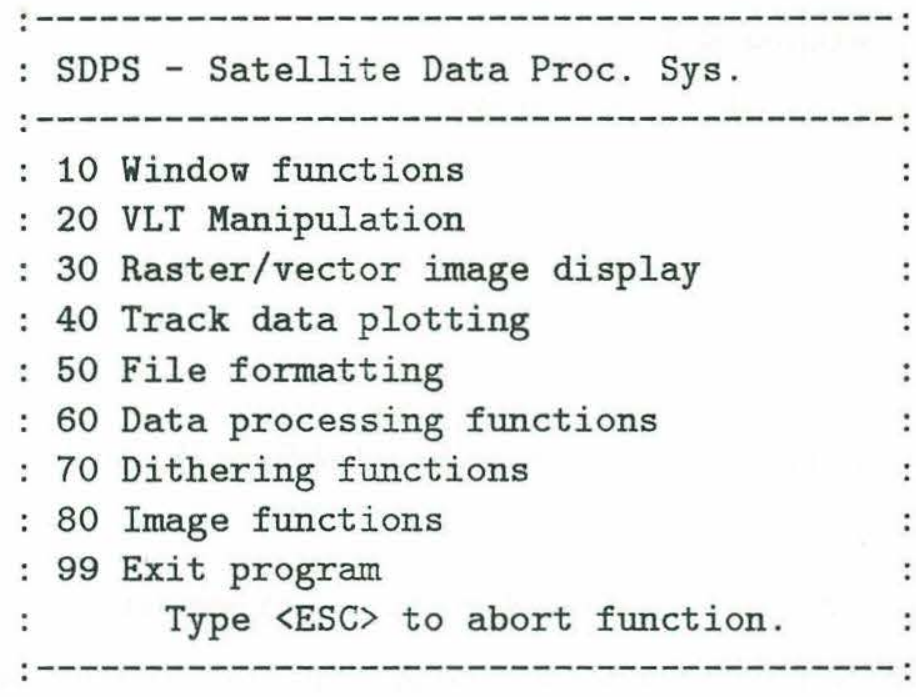

80

Select the Image Functions Submenu

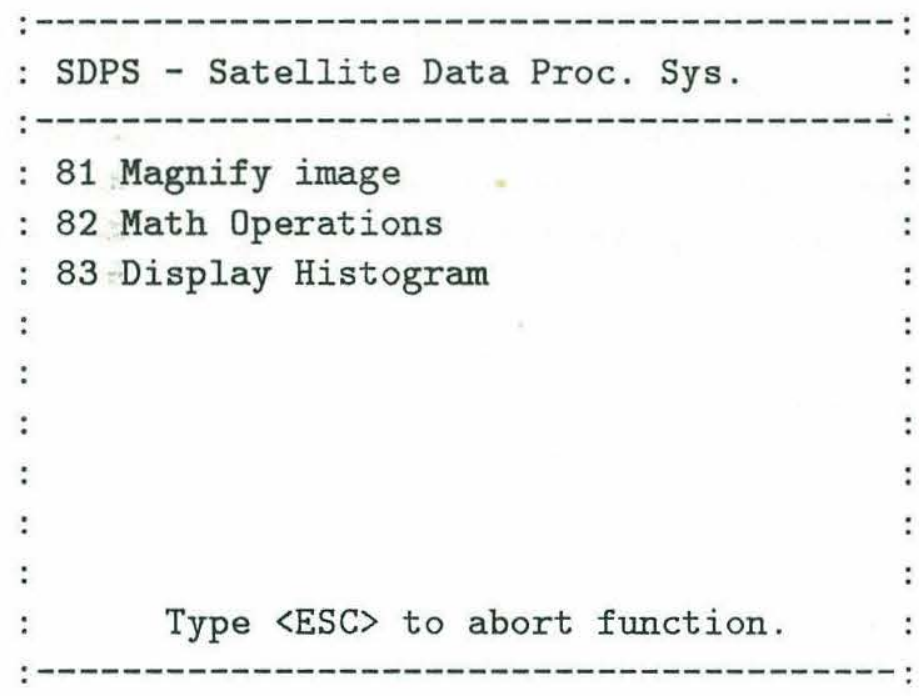

82

Select the Math Operations function

Enter first window \# ?

1

Select the first window to read from 
Enter second memory \#, window \# ?

22

Select the window to put the result

$$
\begin{aligned}
& \text { Enter function ? } \\
& 1 \text { - Add } \\
& 2 \text { - Subtract } \\
& 3 \text { - Multiply } \\
& 4 \text { - Divide }
\end{aligned}
$$

2

The second image will be subtracted from the first

Rescale ? 1 - Yes, 0 - No

0

Keep the same scale used in the second window

The function will be performed and the result will be placed in the second window.

\subsection{Text}

Text is also supported in SDPS. All text input and positioning is done by the user. All text is raster-based and will be output the same as other line-based data (see Basic Output section 9). Text style and size may be selected and examples are shown below. All parameters used for text are in pixels so that the window does not have to be scaled (option 41). An example of writing text to a window is shown:

: SDPS - Satellite Data Proc. Sys.
$: 10$ Window functions
$: 20$ VLT Manipulation
$:$ :
$: 40$ Raster/vector image display
$: 50$ File formatting
$: 60$ Data processing functions
$: 70$ Dithering functions
$: 80$ Image functions
$: 99$ Exit program
: Type <ESC> to abort function.
$:-$




$\begin{array}{lr}\text { : SDPS - Satellite Data Proc. Sys. } & : \\ : \text { : } 41 \text { Set navigation area } & : \\ : 42 \text { Plot navigation lines } & : \\ : 43 \text { Plot Lat/Lon track } \\ : 44 \text { Plot vectors } \\ : 45 \text { Plot Lat/Lon track with scalar value: } \\ : 46 \text { Text annotation } \\ : 47 \text { Line draning via cursor control } \\ : 48 \text { Digitize points } \\ : 49 \text { Plot contours } \\ : \text { Type <ESC> to abort function. } \\ :--\end{array}$

Enter type of font to use:

1) simplex

2) complex

3) italics

4) duplex

Enter font type ?

1 Select the simplex font

Enter text height ?

10

Enter the font height in pixels

Enter text rotation angle ?

0

Text rotation in degrees ccw

Enter value to use (0-255) ?

255

Enter the VLT value to use in drawing (white) 


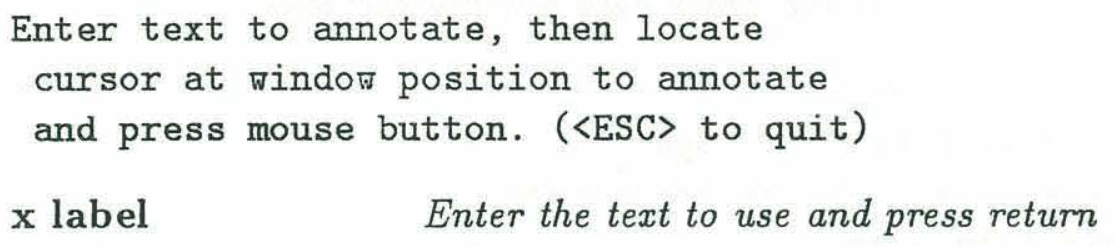

Place the cursor at the lower left hand corner where the text will be placed and press any mouse button. The text will be drawn at that location. Note: pressing the mouse button again will draw the text where the mouse is located. The user may enter new text in the menu window without returning to the main menu and entering the font information again. This can be continued until the user presses the escape key. The length of the text is limited to 41 characters or the width of the menu window. Each subsequent click of the mouse will cause the current text to be written at the cursor location. When new text is entered, it is not put into the write buffer until a $\langle\mathbf{C R}\rangle$ is entered. Note: there is no way to remove text from a window without clearing the entire window.

\section{Basic Output}

SDPS supports two basic types of output, SDPS format and PostScript format. The SDPS format is primarily used for raster-based output while PostScript is generally used for line-based output. SDPS can only generate images of linebased data in SDPS format. SDPS will create a PostScript format file that can be lines or images or both, but is primarily intended to print line drawings on a laser printer.

\subsection{SDPS Format}

This output is used to dump raster-based images from a display window. These images may be loaded later and may be used in most of the external routines (section 12). Two options are available for dumping images from a display window. Option 15 saves the data values in the bitmap including line overlays without CCLM modifications. Option 16 should be used when the changes made to the VLT and should be saved. This option only works for black and white images. To save a remapped color image, the user should first remap the color image using CCLM and then write down the cursor location given in the cursor window that produces the desired result. The user then selects a black and white VLT (option 
21) and remaps the image using CCLM and with cursor location previously written down. Option 16 may then be selected to output the correct image. Option 16 also requests a slope and an intercept. This is optional and simply allows the user to add or change these values in the output header (section 5.4).

\subsection{PostScript}

PostScript may also be used to generate images such as the ones printed in this manual, but with the availability of color hardcopy it is generally used for linebased drawings on a laser printer. Unlike raster output, PostScript uses the higher resolution of the laser printer to print straight lines and smooth curves. Therefore, if you are doing line operations in SDPS, you will want to use the PostScript output option. Option 74 allows the user to generate an image and overlay lines in PostScript format. Option 75 is used when the user wants to create a file containing only the lines.

\subsection{UNIX}

SDPS also allows the user to output line-based data in standard UNIX plot format. This format is described in detail in the UNIX user manuals.

\section{Advanced Functions}

SDPS provides functions for deriving additional information from a given data set. Using SDPS, one can modify a window's VLT to highlight subtle features in an image. The user can also sequentially display up to 15 images to detect time dependent phenomena. SDPS will display data along a line such as a ship track or a satellite ground track, overlay bitmaps to mask an image or draw freehand in a window. The user can also digitize points in an image, such as buoy or drifter locations, to an output file. SDPS also provides support for displaying images on workstations with monochrome screens. The examples given in this section are less detailed than the basic functions section and assumes the user has some familiarity with SDPS. 


\subsection{VLT Modification}

When SDPS initially opens a window, a black and white (greyscale) VLT is automatically loaded in the window (option 21). This VLT is filled from 0 to 255 and when the cursor enters the display window the foreground and background colors used to display text in other windows are temporarily overwritten. This allows the user to use all available colors for displaying an image. Sometimes, the user may want to see the text in external windows (such as the cursor window) or may want to specify certain colors for lines or masks. This can be done by using option 28 to set the image truncation option to $\mathrm{ON}$. When this option is turned $\mathrm{ON}$, all images loaded will be truncated to 253 values instead of 255 . Also, all subsequent VLTs that are loaded will be truncated and location 254 will be loaded with black $(0,0,0)$ and 255 with white $(255,255,255)$. Note: this option does not appear to take effect until a new VLT is loaded (options 21 and 22). If the user desires false color, SDPS supplies 3 distinct false color VLTs. One contains four colors, a second has ten colors, and the third is a 256 color rainbow. If any of these VLTs does not suit the user, a customized VLT may be loaded (option 24). The current VLT may also be customized with option 23 to change 1 to 256 consecutive colors. The entire VLT may also be customized by using Cursor Controlled Linear Mapping (CCLM option 26).

The truncation option may be a source of VLT problems. When the truncation option is set, all subsequent images are truncated. This means that values of 254 and 255 in the image will be set to 253 . If the user wants to have a truncated VLT but not a truncated image first turn the truncation option on, next load in a VLT and turn the truncation option off, finally, load in the image file.

\subsection{Movie Loop}

SDPS may be used to display a sequence of up to 15 images in a time series. Images do not have to be the same size, however, all images are loaded into the upper left hand corner of the selected window and must be in SDPS format. The location of the upper left hand corner cannot be set for individual frames. The following example shows how to load and display five 512 by 512 images with 0.2 seconds separating each frame. The five files are named file.0, file.1, file2., file.3 and file.4. The suffix must be number 0, 1, 2 etc., since SDPS uses this to read the files sequentially. This example assumes that a 512 by 512 display window has been opened. 
Enter filename of SDPS images without \# suffix.

(ie. 'frame.' for frame.0, .1, .2, ...)

file. Enter the base filename of the images

Enter starting memory \# to load ?

1 Start loading in memory location 1

FILE I/O ERROR - file not read

Known bug. Can usually be ignored.

13

This option controls the movie loop

Enter window \# to use ?

1

Select an open window for the display

Enter indices of images $(<=15)$ ?

$12345 \quad$ Order entered is order displayed

Enter delay time (sec) ?

0.2 Select 0.2 seconds between each frame.

Enter number of cycles?

Minimum delay time is limited by the type of machine running SDPS, system load and image size. Speed is also limited because the program does not use hardware or software buffering to keep the program portable. 


\subsection{Plotting Scalar Values Along a Track}

SDPS has an option to plot a scalar value (salinity, sea surface height) along a line such as a ship or satellite ground track. To use this feature, the user must have entered the navigation information using option 41. Assuming the user has a file in SDPS format with the first column containing the latitude, the second the longitude and the third the desired value to plot, the following could be used to plot the data:

Enter window \# to use ?

1

Select the appropriate window

Enter SDPS input file name ?

file.sdps Enter the file name

Enter indices of Lat, Lon, data?

123 Select the correct columns

Enter max expected data value ?

1.0 The data will be scaled $+/-1.0$ units

Enter height from track to display maximum data value ?

20

A data value of 1.0 will be scaled 20 pixels high

Enter value for track, plot (0-255) ?

255255

Both the track and the data will be plotted using color 255

An example of this is shown in figure 5 . 


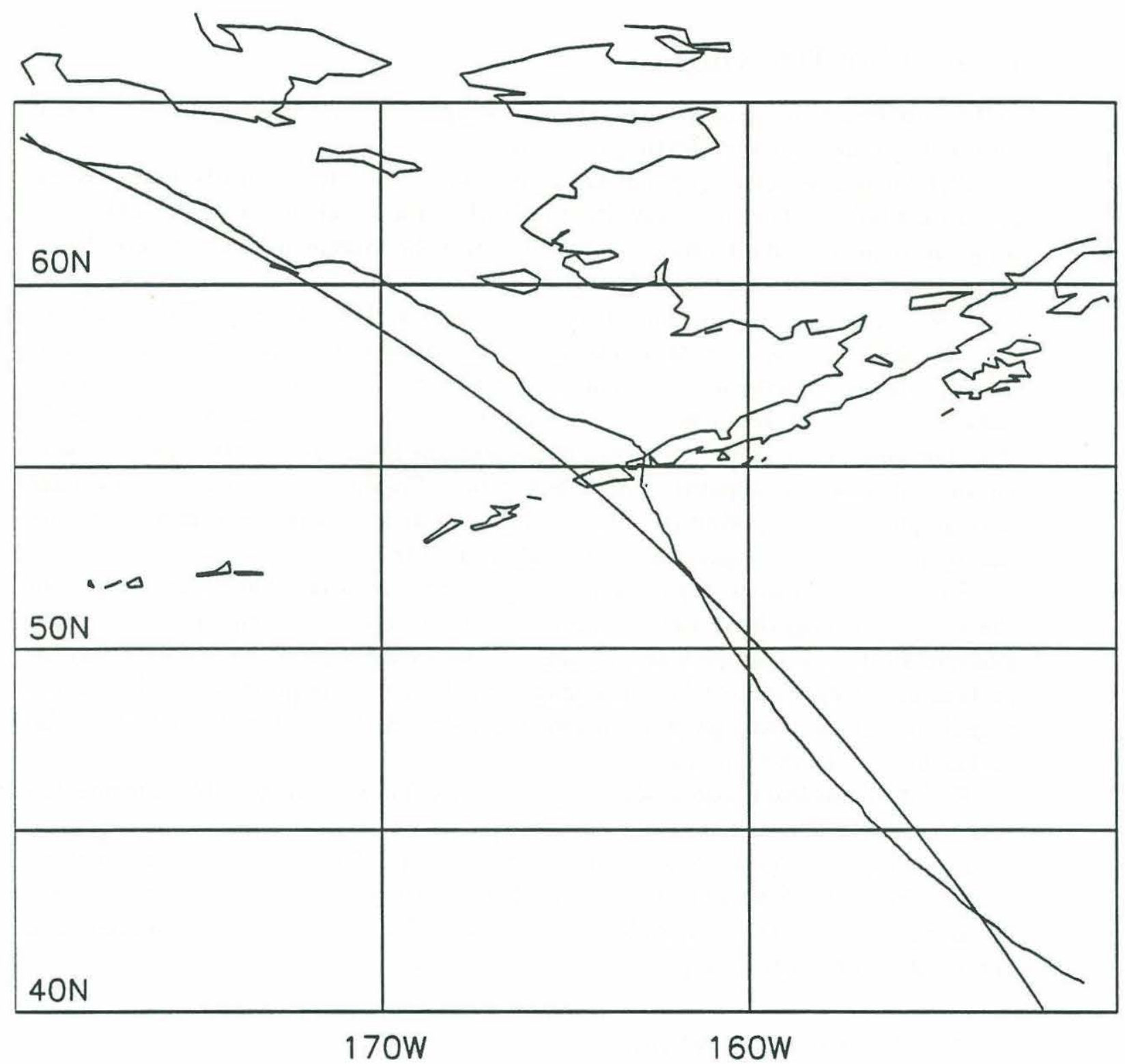

Figure 5: Example of a scalar plot. Coastline was added as a discontinuous line using option 43 . 


\subsection{Line Drawing}

SDPS provides several options for line drawing. All data files must be in SDPS format to be used in the plotting routines.

Plot Navigation Lines (option 42) allows the user to draw a latitude/longitude grid in a window. The user may draw latitude lines, longitude lines or both. The program requires a start value, a stop value and the increment between grid lines. Examples of this are shown in figures 3,4 and 5 .

Plot Lat/Lon track (option 43) is used for most line plotting. This function may be used to draw a continuous line, a discontinuous line or a set of points. The option for drawing a continuous line draws with the pen down line segments between each point in the file. For plotting continuous lines, the user may specify that the input data contain a series of continuous lines each containing the same number of points or separated by a flag value. Finally, each point of a data set may be plotted in the window. These points are plotted using + symbols and the size specified is the number of pixels high and wide.

Plot vectors (option 44) is used to plot arrow vectors. The data may be in the form of a magnitude and a direction or in $u$ and $v$ components. The $u$ and positive 90 degree direction are assumed to be to the right. The vectors may be plotted in a single color where the length of the vector is plotted relative to the magnitude of the data point or where the color of the vector is relative to the magnitude of the data point.

Plot Lat/Lon track with scalar value (option 45) is used for plotting information relative to a lat/lon track.

Line drawing via cursor control (option 47) is used for drawing lines in a display window freehand. Select the first point of the line with the left mouse button and the second point with the middle mouse button. This point may be changed until the right mouse button is pressed to draw the connecting line.

\subsection{Bitmap overlays}

Options 35 and 36 allow the user to overlay bitmaps in an open display window. Option 35 reads an SDPS format file and performs a logical AND on the image previously loaded in the window. All zero values in the mask file will be set to zero in the image. All other values in the mask image will not affect the image displayed. Option 36 is similar except that a value of zero in the mask image will not affect the image displayed. All other values in the bitmap image will replace 
the corresponding values in the display. The options are useful for masking out land values.

\subsection{Digitize Points}

When a display window has a feature such as a front that the user would like to digitize, SDPS will digitize the cursor location and output the information to a file. If the navigation information has been set, the output values will be in latitude-longitude coordinates.

\subsection{Dithered Images}

For users with monochrome workstations, most line-based functions will work. When an option asks for a VLT color, select 0 or 255 for black or white. Image and VLT functions will not work however, images may be displayed using the dithering options. Binary image files and SDPS format files can be displayed on a monochrome monitor using options 71 and 72 . These functions display the image using a 4 by 4 dither to provide 17 different patterns (including black and white). Input parameters are the same as for options 32 and 33.

\subsection{Defaults}

Many functions include default or commonly used values. This values are listed in square brackets [] where the default is available. To select the default values, simply press return at the prompt.

\section{Menu Listings and Descriptions}

\subsection{Window Functions}

\section{OPEN WINDOW}

This function opens a window for displaying raster images or line plots. The size of the window is specified (in pixels) by the user. Currently, a window should not be resized after it is opened, as this will adversely effect the operation of other functions on this window. Preferably, the user should select QUIT from the window and open a new window if a different size is needed. 


\section{REDISPLAY IMAGE}

This function displays the raster information previously stored in memory by the user. This may be an image stored using option 14 or a memory location chosen when a file was initially loaded.

\section{FLICK BETWEEN IMAGES}

This function displays a sequence of images previously stored in memory by the user individually using option $14,31,32$ or 33 , or sequentially using option 34. The user is prompted for the window to use, the delay time in seconds between frames and the indices of the memory locations to use. Currently, up to fifteen frames may be displayed.

\section{OUTPUT WINDOW TO MEMORY}

This function allows the user to store a display window in memory as a raster image. Typically this is used when non-raster data such as line plotting or text annotation is plotted and the user would like to save it as a raster image to redisplay at a later time. This is also useful when a window is needed for an additional display or when the user wants to insert data into specific memory locations for flickering using option 13.

\section{OUTPUT WINDOW TO FILE}

This function saves the data displayed in a window to a file as a raster image. The image file contains the actual data values in the bitmap. Changes in the VLT such as those done by CCLM (Cursor Controlled Linear Mapping) will not effect the saved image. Changes made to the bitmap such as notation and lines will be saved. To save changes made to the displayed intensities refer to option 16.

\section{OUTPUT B\&W REMAPPED WINDOW TO FILE}

This function saves the intensity values displayed in a window in SDPS file format. This function differs from the previous one because it uses the current VLT of the window to remap the data before it is output. This allows the user to display a black and white image, change the VLT with CCLM (Cursor Controlled Linear Mapping) and save the enhanced image. This option prompts for a slope and an intercept for the output image. Where intercept $=$ (true value) corresponding to a screen byte value of zero and the slope $=$ (true value - intercept $) / 255$. By just pressing return at this prompt, you will keep the current value of the window. 


\section{CLEAR WINDOW}

This function clears the user-specified window by entering zero (0) into all the pixels in the window bitmap. It does not clear any corresponding image in memory. The default value for the window number will be the last window accessed.

\section{DISPLAY COLORBAR}

This function displays a colorbar for a specified window. If the image in that window was loaded as an SDPS format file and the header for that file has the slope and intercept set (section 5.4 ), the true values will be displayed along the colorbar. Note: that the colorbar displays colors in the current VLT.

\section{DUMP WINDOW TO UNIX PLOT FILE}

This function allows the user to output the lines displayed in a window to a unix plot file ${ }^{6}$. This file may then be displayed with any routine used to display standard unix plot files.

\subsection{VLT Manipulation}

\section{LOAD BLACK/WHITE VLT}

This function loads a grey scale VLT (Video Lookup Table) into the user specified window. The VLT is a linear function with a slope of 1 and an intercept of 0 . The VLT is pure black $(0,0,0)$ for pixel values of 0 and pure white for pixel values of 255 . If the auto-truncation option is on, pure white $(255,255,255)$ is truncated at 253 and 254 is reserved for black while 255 is reserved for white.

\section{LOAD COLOR VLT}

This function loads a pseudo-color VLT for the user specified window. The user has a choice of a 4,10 , or 256 color VLT. The four color VLT provides four distinct colors: blue, green, yellow or red. The ten color VLT provides the following distinct colors: purple, midnight blue, dark blue, light blue, dark green, light green, yellow, orange, red and dark red. The 256 color VLT provides the same basic colors as the 10 color VLT, but also provides intermediate shades.

\footnotetext{
${ }^{6}$ See UNIX manual for more information on plot file format
} 


\section{LOAD INDIVIDUAL VLT VALUES}

This function allows the user to change one or more VLT values to any user specified RGB value. This is useful when a user wishes to highlight a feature that is represented by a single value, such as to give navigation lines and coastlines different colors when plotting. The values for red, green and blue should range from $0,0,0$ (black) to $255,255,255$ (white). The user must supply the VLT starting position and the number of values to change. These values should vary from 0 to 255 and the total should be less than 256 . The user should have some familiarity with RGB color concepts.

\section{READ VLT FROM FILE}

This function reads a binary file of $256^{*} 3$ byte values to specify the VLT of a window. SDPS does not support ASCII files. This function is primarily intended to read a VLT written by the option 25 . The user may create a VLT by writing a binary file with 256 byte values for red followed by 256 byte values for green and 256 byte values for blue.

25 WRITE VLT TO FILE

This function writes the VLT from a user specified window to a file. This file may be read later using option 24. This is useful for saving VLT modified using option 26.

26 CURSOR CONTROLLED LINEAR MAPPING

This function uses the cursor to control a linear VLT mapping function. The cursor position is read and the VLT for the window specified is remapped to another VLT which is then used for display. The vertical position of the cursor determines the slope of the function and the horizontal position determines intercept. Hence with the cursor in the middle of the window the slope is zero, at the ends it is infinity, and three quarters up from the bottom it is one, and one quarter up from the bottom it is negative one. With the cursor in the middle an old VLT value of 128 is mapped to 128 . With the cursor to the left it is mapped to 0 , and with the cursor to the right it is mapped to 255 . Note: the entire image is used for the mapping not just the view window.

\section{INVERT VLT VALUES}

This function inverts the values of the VLT for a window so that the color for 0 is now used for 255 , and the color for 255 is now used for 0 . This changes 
the sign of the slope of the mapping function but not the magnitude. If the truncation option is set, only the first 253 values are inverted.

\section{SET AUTO-TRUNCATION OPTION}

This option when set to ON, will automatically truncate values of input raster images to the $0-253$ range, setting values of 255 and 254 to 253 . Also, the VLTs that are placed into windows or assigned at window creation will reserve 254 and 255 for black and white respectively. This allows the other SUNVIEW windows to be viewed simultaneously with the display windows. To preserve values of 254 and 255, set auto-truncation OFF and then load the image. If black and white values are needed for the VLT locations 254 and 255, then turn auto-truncation ON and load in a VLT. This will give an untruncated image with a truncated VLT. This function cannot be specified separately for each window. Once specified it will remain in effect for all windows until turned OFF. VLTs will not be affected by this option until a new VLT is loaded.

\section{SET CURSOR COLOR}

This option allows the user to change the color of the cursor. It is sometimes necessary to change the cursor color in a window to distinguish it from the image. The byte value (from 0 to 255 ) is set to the corresponding color in the window's VLT.

\subsection{Raster Image Display}

\section{DISPLAY SUN RASTERFILE}

This function reads a SUN rasterfile, which contains a header, an optional VLT and an image, and displays it in the user specified window. The last window opened is both the default window number and the default memory number. The default display position is row zero and column zero which corresponds to the upper left hand corner.

\section{DISPLAY BINARY IMAGE FILE}

This function reads a file containing an optional header and a byte image of any size and displays the image in the specified window. Here, the default memory number, window number and display position are the same as for option 31. The default image size corresponds to the image size used when opening the window (see option 11). The default number of header bytes to 
skip is zero. If header bytes are not skipped, they will be displayed as part of the image.

\section{DISPLAY SDPS IMAGE FILE}

This function reads a file in SDPS file format and displays the 2D data as a raster image in the specified window. This function currently only reads byte files. For information on converting file formats see sections 5 and 12.2. The defaults used here for memory number, window number and display position are the same as for options 31 and 32 .

\section{READ MULTIPLE SDPS IMAGE FILES}

This function reads multiple SDPS format files into memory to be displayed later. These files must have $.0, .1, .2, \ldots$ as suffixes in order to be read in correct order. This function is typically used with multiple files which are viewed with option 13 in a movie-loop fashion. The images are stored in consecutive memory positions starting at the specified memory position.

35 DISPLAY SDPS MASK FILE

This function reads an SDPS file and uses it to mask an image previously loaded in a window. This is done by performing a logical $A N D$ of the image file read in with the image in the display window. Locations with 0 in the mask file will be set to zero, while values of 255 will not affect the image in the display window.

\section{OVERLAY SDPS BIT MAP}

This function is similar to 35 except that it places the value of the bitmap file in the window for all values greater than 0 . A bitmap value of 0 will not be overlaid on the window.

\subsection{Track Data Plotting}

\section{SET NAVIGATION AREA}

This function sets the coordinate system for line plotting in the display windows and must be used before any plotting is attempted. There are three map projections to chose from: Stereographic, Mercator, and Equirectangular. Each projection has three parameters to describe the size and orientation. The first is center latitude and longitude, which defines the latitude and longitude at the point in the center of the window. Second is rotation, 
which defines rotation of the coordinate system about the center point in the window. Thirdly, $\mathrm{x}$ and $\mathrm{y}$ scale factors determine the scale in the horizontal and vertical direction of the window.

For the Stereographic projection, a scale of one in each direction allows the zero latitude line to fit just within the boundaries of the window. With a scale of one for the Mercator projection, 360 degrees of longitude can fit horizontally in the window and just above and below positive and negative 80 degrees latitude. The Equirectangular projection allows an even proportion of degrees per distance in both the latitude and longitude directions, and so 360 degrees will fit both horizontally and vertically in the window with $\mathrm{x}$ and $\mathrm{y}$ scale factors of one.

\section{DRAW NAVIGATION LINES}

This function allows the user to draw latitude and longitude lines in a specified window given a minimum, maximum and incremental value for latitude and longitude. The user may chose a VLT value between 0 and 255 to use for writing to the window. The parameters in option 41 must be set before using this option.

\section{PLOT TRACK}

This function reads an SDPS format file containing latitude and longitude coordinates. The input data may represent a continuous line, a discontinuous line or a set of individual points. If the continuous line option is chosen, the program will draw all points connected with a single continuous line. It will attempt to break the line if it crosses the window boundary. There are two types of discontinuous lines available. The user may input a data set that is broken by a specified delimeter or a data set that contains segments of equal length. The user may also choose to input a data set that contains discrete points. The program plots these points using a small plus sign. The data is assumed to be in columnar format where each row contains a latitude coordinate, a longitude coordinate and optionally any other data such as the magnitude and direction to be used in a vector plot. In order to use an ASCII file for this option, the user must first convert to SDPS format using option 51. This converts the file to binary for faster reading/writing. The user may choose a VLT value between 0 and 255 to use for writing to the window. 


\section{PLOT VECTORS}

This function reads an SDPS format file and plots magnitude and direction in the form of variable length arrows. The positional coordinates must be latitude and longitude. The vector may be defined as $\mathrm{x}$ and $\mathrm{y}$ components or as magnitude and direction. The user may chose a value between 0 and 255 to use for writing to the window. $\mathrm{X}$ is assumed to be positive to the right and y is positive to the top. Direction is given as positive 90 degrees to the right and 0 degrees to the top.

\section{PLOT SCALAR VALUES ALONG TRACK}

This function reads an SDPS format file and plots a latitude, longitude track and a line with the $\mathrm{x}$-axis parallel to the track and the $\mathrm{y}$-axis perpendicular to the track. The user is then asked for a maximum expected value. A positive value is interpreted as the absolute value of the data and is used to scale the data. If a negative value is selected, the program finds the maximum absolute value of the data, uses it to scale all the data and prints the maximum on the screen. If this value is less than the maximum encountered by the program, the actual maximum will be displayed. The user is then prompted for the height from the track to display maximum value, i.e., the number of pixels between the latitude-longitude track and the maximum value to be plotted. The user then choses separate values between 0 and 255 for plotting the latitude-longitude track and the line.

\section{TEXT ANNOTATION}

This function reads an input character string and outputs it to a user specified location in a display window.

\section{LINE DRAWING VIA CURSOR CONTROL}

This function allows the user to input straight lines to a window using the cursor. Clicking the left mouse button locates the starting point, the middle mouse button locates the end point, and clicking right mouse button draws the line. The left and middle mouse buttons echo their position when pressed.

\section{ECHO CURSOR LOCATION}

This function prints the cursor position in a display window when the left mouse button is clicked. This function prints the cursor values to the menu window and to a file specified by the user. If option 41 has set the navigation 
parameters, the output will be in lat-lon coordinates. Otherwise, the row and column values are printed.

\section{PLOT CONTOURS}

This function plots selected contours from an SDPS format file. The file must be rectangular, but does not need to have even spacing. The user may specify a mask file for areas that should not be contoured. This file is optional and if it is not specified, the default is to use all data points in the input file. The latitude and longitude are input with one dimensional SDPS format files. Each entry in the longitude file correspond to each data point from left to right and each entry in the latitude file correspond to each data point from top to bottom. The user may select a range of contour levels and the increment between each level. The user may also select a range of colors to plot each contour level from 0 to 255 . To select a single color, enter the number twice.Note: The input file, the longitude and latitude files are floating point format files and the mask file is a byte type file.

\subsection{File Reformatting}

\section{REFORMAT ASCII TO SDPS}

This function reads an ASCII file with a fixed number of columns of data and converts it to an SDPS format file of two dimensions. A more flexible and robust method of converting files from ASCII to SDPS is done in the external routine sdps_convert (section 12.2).

\section{REFORMAT BINARY IMAGE TO SDPS}

This function read a binary image file and strips off header information, then stores it as an SDPS format file of two dimensions. This option does not set the slope and intercept values. These may be set in the external subroutine sdps_headedit (section 12.7).

\subsection{Data Processing Functions}

\section{CONVERT EAST/NORTH TO LAT./LON.}

This function reads a format file containing $\mathrm{N}$ columns of data with two columns of east and north distances in kilometers, and using a basepoint latitude, longitude position, changes the position data to latitude, longitude 
coordinates. The result is then written to an SDPS format file of the same size and number of columns. This option may be removed in future versions.

\subsection{Dither And PostScript Functions}

\section{DISPLAY DITHERED IMAGE}

This function is similar to option 32 . It is used to display a binary image file on a monochrome monitor. The image is displayed by dithering a 4 by 4 pixel area using 17 different patterns (including black and white). The average of the 4 by 4 area is used to determine which dither pattern to display. Since some SDPS functions do not work on monochrome monitors, images loaded using this function are useful only for previewing images.

\section{DISPLAY DITHERED SDPS}

This function is similar to option 71 except that it dithers SDPS format files.

\section{DISPLAY DITHER BAR}

This function opens a new window and displays the different dither patterns. It does not display the pixel values like option 18 .

\section{DUMP PS SDPS IMAGE}

This function reads an SDPS format file from disk and formats the output in a PostScript-compatible file. This function optionally overlay lines from a display window. The image file is read from disk and not the window so that overlaid lines will not be dithered with the image. There is also an optional colorbar that displays the values associated with ten different greyscales. These values will only be displayed correctly if the input file has the slope and intercept set in the header (section 5.4).

\section{DUMP LINES TO PS FILE}

This function reads the lines and vectors from a display window and writes them to a PostScript file. This is an ASCII file and may be edited for special effects or may be sent directly to a PostScript printer.

\subsection{Image Functions}

\section{MAGNIFY IMAGE}

This function allows the user to read an image from one window, magnify 
it and put it in a second window. The user may choose from two types of magnification: simple pixel replication and bi-linear interpolation. The simple pixel replication technique allows the user to specify a magnification factor in each of the row and column directions. The bi-linear interpolation technique only accepts magnification factors that are powers of 2 and does not allow for separate row and column factors. The bi-linear technique will produce a smoother magnified image, but will also blur sharp features such as coastlines.

\section{MATH FUNCTIONS}

This function allows the user to add, subtract, multiply or divide one image from/by another. The result is placed in the second window. The images loaded in the two display windows must be in SDPS format and must have the slope and intercept specified. This option converts back to real before performing the operation and then converts back to byte before loading the results back into the display window. All operations are done on the image in the first window by the image in the second window and are displayed in the second window. For example: $\operatorname{image} 2(i, j)=\operatorname{image1}(i, j)-\operatorname{image} 2(i, j)^{7}$.

\section{HISTOGRAM}

This function opens a new window and displays the histogram of a specified display window. The color of each bar corresponds to the same color pixels in the display window. Note: this function only works with color monitors and is not automatically updated when the window contents change.

\section{External Utilities}

This section describes various utilities that may be used to perform image manipulation without invoking SDPS.

\section{1 sdps_append}

This program is used to append two SDPS format files together. Usage: sdps_append row_off col_off file1.sdps file2.sdps > file3.sdps

\footnotetext{
${ }^{7}$ See section 5.4 for more information on SDPS file types
} 
The first file is located at 0,0 and the second file is located at integer location row_off, col_off as specified on the command line. Col_off is the offset along a row and row_off is the offset along a column. File3.sdps is a new SDPS format file that is col_off plus the number of columns in file2.sdps wide and row_off plus the number of rows in file2.sdps high. Note: that the upper left hand corner of file2.sdps and file3.sdps is located at 0,0. Therefore if file1.sdps has 200 rows and 300 columns and file2.sdps has 300 rows and 100 columns, the following command would be used to append file2.sdps to the right of file1.sdps:

sdps_append 2990 file1.sdps file2.sdps > file3.sdps

while the following command appends file2.sdps to the bottom of file1.sdps:

sdps_append 0199 file1.sdps file2.sdps > file3.sdps

File3.sdps would have 300 rows and 400 columns in the first example and 500 rows and 300 columns. Any location not filled from file1.sdps or file2.sdps would be filled with a 0 .

\section{2 sdps_convert}

This program is used to convert ASCII files to SDPS floating point files. Usage:

sdps_convert rows cols [drow dcol] < file1.asc > file2.sdpsf

The ASCII file is assumed to be free format with at least rows times cols data points. The program checks for an end-of-file so that the argument rows may be larger than the actual number of rows in the input file. The program also assumes the data to be rectangular and ordered from left to right, top to bottom with the number of columns known. The user may optionally enter the row and column spacing using the input parameters drow and $d c o l$. These values are used in other auxiliary programs. For more information on drow and dcol see SDPS format files (section 5.4). For example, if file1.sdps contains the following ASCII latitude-longitude ship locations: 
10.0290 .0

$10.25 \quad 292.35$

10.51293 .61

the following command would be used to convert line data to an SDPS float format file:

sdps_convert $32<$ file1.asc > file2.sdpsf

File2.sdpsf could now be used in SDPS to identify the ship tracks.

\section{3 sdps_cut}

This program cuts a section from an SDPS format file. Usage:

sdps_cut rowul colul rowlr collr < file1.sdps > file2.sdps

The program reads file1.sdps, cuts the section from rowul, colul to rowlr, collr and writes the resulting file2. The program keeps the original slope and intercept header values from file1.sdps so that file2 will use the same scale. The only modification to the output header file is to change the size. Note: the upper left corner of the data file is located at 0,0 . To cut the upper left 256 by 256 section from a 512 by 512 file, the following command would be used:

sdps_cut $00255255<$ file1.sdps > file2.sdps

\section{4 sdps_flip}

This program flips an SDPS file around a row or column axis. Usage:

sdps_flip dir < file1.sdps > file2.sdps

If $\operatorname{dir}$ is 1 , the program flips the file around the image from top to bottom. If dir is 2 , the program flips the file around from left to right. For example:

sdps_flip $1<$ file1.sdps > file2.sdps

Makes the top row of file1.sdps the bottom row in file2.sdps. 


\section{5 sdps_ftb}

This subroutine reads SDPS float format files and converts them to byte format files. Usage:

sdps_ftb [-mxlhf] [min max low high] < file1.sdpsf > file2.sdps

Each point is scaled between the $\max$ and $\min$ to the range of byte values $(0-253$ for truncated and 0-255 for full files). The options specify how to perform the scaling:

$\begin{array}{ll}\text { Option } & \text { Description } \\ -\mathrm{m} & \text { Use the user specified value as minimum for scaling. } \\ -\mathrm{x} & \text { Use the user specified value as maximum for scaling. } \\ -\mathrm{l} & \text { Values }<\min \text { are specified by user (use with }-\mathrm{m} \text { ) } \\ \text {-h } & \text { Values }>\max \text { are specified by user (use with }-\mathrm{x} \text { ) } \\ -\mathrm{f} & \text { Use all } 256 \text { byte values for scaling the output }\end{array}$

The values for min and max are floating point and low and high are integer (0 255). Note: that the order of the options must correspond to the order of the variables and the options must precede the variables: $-x m \max \min$ is acceptable, but $-m \min -x \max$ is not. By default, the output file values are truncated ( 0 -253) with 254 and 255 reserved for black and white.

To create a truncated byte image with 0 as the minimum and 253 as the maximum of the floating point image:

sdps_ftb $<$ file1.sdpsf > file2.sdps

File2.sdps will be an SDPS byte image.

If a floating point file has a -999.0 mask flag, a maximum of 20.0, minimum of -10.0 and the user wants to mask the -999.0 values with black on the byte image file:

sdps_ftb -mxlh $-10.020 .0254254<$ file1.sdpsf > file2.sdps.

File2.sdps will be scaled to a minimum of -10.0 and a maximum of 20.0. All values less than -10.0 or greater than 20.0 will be set to 254 (black) 


\section{6 sdps_header}

This program displays the header from an SDPS format file. Usage:

sdps_header < file1.sdps

\section{7 sdps_headedit}

This program edits the header from an SDPS format file. Usage:

sdps_headedit file1.sdps > file2.sdps

The program displays the current value of each header value and queries the user for a new value. Pressing return retains the old values.

\section{8 sdps_logscale}

This program rescales an SDPS float file to a log scale. Usage:

sdps_logscale < file1.sdpsf > file2.sdpsf

This program amplifies an SDPS float format file (such as a magnitude file). The program assumes that the input file only contains positive numbers. If negative numbers are found, the program prints an error message and exits. This program only works on SDPS float format files.

\section{9 sdps_mask}

This program masks one SDPS format file with another. Usage:

sdps_mask file1.sdpsf file2.sdps threshold [mask_value] > file3.sdpsf

The program reads file1.sdpsf as the input file to be masked and file2.sdps as the mask file. Threshold is the value to compare against the mask file. If the mask file is less than the threshold value, then the original point in file1.sdps is left intact. If the value in the mask file is greater than or equal to the threshold value, the original point is changed to zero or to the optional mask_value. This program copies the header from file1.sdps to file3.sdpsf and does not modify the slope or the intercept. The program masks the section beginning in the upper left hand corner of the original image. The mask image must be less than or equal to the size of the image to be masked. File1.sdps is a float type file, file2.sdps is a byte type file, threshold is an integer and mask_value is a floating point value. 


\subsection{0 sdps_math}

This program adds, subtracts, multiplies or divides two SDPS format files together. Usage:

sdps_math op file1.sdpsf file2.sdpsf > file3.sdpsf

The math operation $o p$ is performed by file2.sdpsf on file1.sdpsf and the result is placed in file3.sdpsf. The option op indicates which operation to perform:

$\begin{array}{ll}\text { Option } & \text { Operation } \\ \text { a } & \text { Add file2.sdpsf to file1.sdpsf. } \\ \text { s } & \text { Subtract file2.sdpsf from file1.sdpsf. } \\ \text { m } & \text { Multiply file1.sdpsf with file2.sdpsf. } \\ \text { d } & \text { Divide file1.sdpsf by file2.sdpsf. }\end{array}$

\subsection{1 sdps_pad}

This program pads one or more edges of an SDPS format file with a specified value or with the average value of the image. Usage:

sdps_pad flags pad_size pad_val < file1.sdpsf > file2.sdpsf

This program places the value given in pad_val around the edges specified in the flags argument. The number of points added to each edge is the integer value pad_size. If pad_val is an ASCII character, the mean of the image will be used for padding. The argument flags is used to specify how to pad the image:

$\begin{array}{ll}\text { Option } & \text { Description } \\ l & \text { Pads left edge of image. } \\ r & \text { Pads right edge of image. } \\ t & \text { Pads top edge of image. } \\ b & \text { Pads bottom edge of image. } \\ \text { a } & \text { Pads all edges of image. }\end{array}$

Flags can be combined to pad more than one edge. The flags lrtb are the same as the single flag $a$. Note: that flag $a$ should be used alone. To pad an SDPS format file on the top and right edge with the average value of the image: 
sdps_pad tr 1 a $<$ file1.sdpsf > file2.sdpsf

To pad this image with 2 pixels of 0.0 around all edges:

sdps_pad a $20.0<$ file1.sdpsf > file2.sdpsf

\subsection{2 sdps_rotate}

This program rotates an SDPS format file around the origin. Usage:

sdps_rotate deg < file1.sdps > file2.sdps

The command line argument deg must be 90,180 or 270 . A positive number indicates rotation in the counter-clockwise direction, while a negative number indicates rotation in the clockwise-direction. To rotate an image 90 degrees counter clockwise:

sdps_rotate $90<$ file1.sdps $>$ file2.sdps

\subsection{3 sdps_to_sun}

This program converts an SDPS format image file to a SUN rasterfile. Usage:

sdps_to_sun [-ac vltname] < file1.sdps > file2.sun

By default, it uses the same truncated full color VLT that SDPS uses. This assigns 254 to black and 255 to white. The user may optionally specify an ASCII VLT with values of red, green and blue on a separate line. The program expects to read 256 rows of colors. If these data are in file vlt.asc, then the following will use that VLT:

sdps_to_sun -a vlt.asc $<$ file1.sdps $>$ file2.sun

The user may also specify a binary VLT as output using option 25 in SDPS. If this VLT is in file vlt.sdps, then the following will convert an SDPS image to a SUN rasterfile:

sdps_to_sun -c vlt.sdps < file1.sdps > file2.sun 


\section{Acknowledgements}

The authors would like to thank Drs. Nick Fofonoff, Hans Graber and Kathryn Kelly for their support and advice in this project, Carol Alessi, Roger Goldsmith, and Debbie Barber for their suggestions on the content of this manual and Derek Spencer for providing additional funding for the Remote Sensing Analysis Laboratory. 


\section{A Example ASCII Line data file}

27.600000286 .670013

27.629999287 .779999

27.690001288 .579987

27.860001289 .309998

28.129999290 .070007

28.360001290 .720001

28.620001291 .359985

28.920000291 .920013

29.500000292 .510010

$29.850000 \quad 292.829987$

30.260000293 .209991

$31.170000 \quad 293.649994$

32.020000294 .179993

32.779999294 .480011

33.599998294 .890015

\section{B Example ASCII Vector data file}

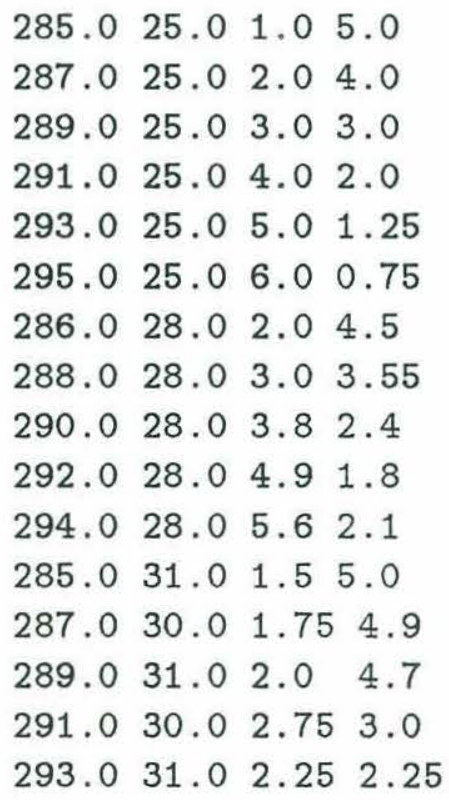


$\begin{array}{lllll}295.0 & 30.0 & 2.0 & 1.75\end{array}$

$\begin{array}{lllll}285.0 & 34.0 & 1.5 & 5.3\end{array}$

$287.0 \quad 34.0 \quad 1.75 \quad 5.2$

$290.0 \quad 34.0 \quad 2.0 \quad 5.1$

$292.0 \quad 34.0 \quad 2.25 \quad 4.0$

$295.0 \quad 34.0 \quad 2.5 \quad 4.0$ 


\section{DOCUMENT LIBRARY}

May 5, 1989

\section{Distribution List for Technical Report Exchange}

Attn: Stella Sanchez-Wade

Documents Section

Scripps Institution of Oceanography

Library, Mail Code C-075C

La Jolla, CA 92093

Hancock Library of Biology \& Oceanography

Alan Hancock Laboratory

University of Southern California

University Park

Los Angeles, CA 90089-0371

Gifts \& Exchanges

Library

Bedford Institute of Oceanography

P.O. Box 1006

Dartmouth, NS, B2Y 4A2, CANADA

Office of the International

Ice Patrol

c/o Coast Guard R \& D Center

Avery Point

Groton, CT 06340

Library

Physical Oceanographic Laboratory

Nova University

8000 N. Ocean Drive

Dania, FL 33304

NOAA/NESDIS Miami Library Center 4301 Rickenbacker Causeway

Miami, FL 33149

\section{Library}

Skidaway Institute of Oceanography

P.O. Box 13687

Savannah, GA 31416

Institute of Geophysics

University of Hawaii

Library Room 252

2525 Correa Road

Honolulu, HI 96822

Library

Chesapeake Bay Institute

4800 Atwell Road

Shady Side, MD 20876

MIT Libraries

Serial Journal Room 14E-210

Cambridge, MA 02139
Director, Ralph M. Parsons Laboratory

Room 48-311

MIT

Cambridge, MA 02139

Marine Resources Information Center

Building E38-320

MIT

Cambridge, MA 02139

Library

Lamont-Doherty Geological

Observatory

Colombia University

Palisades, NY 10964

Library

Serials Department

Oregon State University

Corvallis, OR 97331

Pell Marine Science Library

University of Rhode Island

Narragansett Bay Campus

Narragansett, RI 02882

Working Collection

Texas A\&M University

Dept. of Oceanography

College Station, TX 77843

Library

Virginia Institute of Marine Science

Gloucester Point, VA 23062

Fisheries-Oceanography Library

151 Oceanography Teaching Bldg.

University of Washington

Seattle, WA 98195

Library

R.S.M.A.S.

University of Miami

4600 Rickenbacker Causeway

Miami, FL 33149

Maury Oceanographic Library

Naval Oceanographic Office

Bay St. Louis

NSTL, MS 39522-5001

Marine Sciences Collection

Mayaguez Campus Library

University of Puerto Rico

Mayagues, Puerto Rico 00708 



\begin{tabular}{|c|c|c|c|}
\hline $\begin{array}{l}\text { REPORT DOCUMENTATION } \\
\text { PAGE }\end{array}$ & $\begin{array}{l}\text { 1. REPORT NO. } \\
\text { WHOI-89-13 }\end{array}$ & 2. & 3. Recipient's Accession No. \\
\hline \multirow{2}{*}{\multicolumn{3}{|c|}{$\begin{array}{l}\text { 4. Title and Subtitle } \\
\text { Satellite Data Processing System (SDPS) Users Manual V1.0 }\end{array}$}} & $\begin{array}{r}\text { 5. Report Date } \\
\text { May } 1989\end{array}$ \\
\hline & & & 6. \\
\hline \multicolumn{3}{|l|}{$\begin{array}{l}\text { 7. Author(s) } \\
\text { Michael Caruso, Chris Dunn }\end{array}$} & $\begin{array}{l}\text { 8. Performing Organization Rept. No. } \\
\text { WHOI-89-13 }\end{array}$ \\
\hline \multirow{2}{*}{\multicolumn{2}{|c|}{$\begin{array}{l}\text { 9. Performing Organization Name and Address } \\
\text { The Woods Hole Oceanographic Institution } \\
\text { Woods Hole, Massachusetts } 02543\end{array}$}} & & 10. Project/Task/Work Unit No. \\
\hline & & & $\begin{array}{l}\text { 11. Contract(C) or Grant(G) No. } \\
\text { (C) N00014-86-K-0751; } 957652 \\
\text { (G) }\end{array}$ \\
\hline \multirow{3}{*}{\multicolumn{2}{|c|}{$\begin{array}{l}\text { 12. Sponsoring Organization Name and Address } \\
\text { The Office of Naval Research; and the National Aeronau- } \\
\text { tic and Space Administration }\end{array}$}} & & 13. Type of Report \& Period Covered \\
\hline & & & Technical Report \\
\hline & & & 14. \\
\hline
\end{tabular}

15. Supplementary Notes

This report should be cited as: Woods Hole Oceanog. Inst. Tech. Rept., WHOI-89-13.

16. Abstract (Limit: 200 words)

SDPS was written to allow users to quickly and easily display image and line-based data on inexpensive Sun workstations. The primary purpose of the program is for assimilating oceanograpnic data that may be generated from a satellite, a ship or a buoy. This manual describes the various options available and examples of how to use them. Also discussed are several additional programs for managing data external to SDPS.

\section{Document Analysis a. Descriptors}

1. satellite

2. graphics

3. image processing

b. Identifiers/Open-Ended Terms

c. COSATI Field/Group

\begin{tabular}{|c|c|c|}
\hline \multirow{2}{*}{ Approved for publication; distribution unlimited. } & $\begin{array}{l}\text { 19. Security Class (This Report) } \\
\text { UNCLASSIFIED }\end{array}$ & $\begin{array}{l}\text { 21. No. of Pages } \\
58\end{array}$ \\
\hline & 20. Security Class (This Page) & 22. Price \\
\hline See ANSI-Z39.18) & ierse & $\begin{array}{l}\text { OPTIONAL FORM } 272 \text { (4-77) } \\
\text { (Formerly NTIS-35) } \\
\text { Department of Commerce }\end{array}$ \\
\hline
\end{tabular}


